\title{
Exploring the Mechanisms Underlying the
}

Therapeutic Effect of Salvia Miltiorrhiza Against Diabetic Nephropathy Using Network Pharmacology
and Molecular Docking

\section{Li-Li Zhang}

China Academy of Chinese Medical Sciences Guanganmen Hospital

Lin Han

China Academy of Chinese Medical Sciences Guanganmen Hospital

\section{Xin-Miao Wang}

China Academy of Chinese Medical Sciences Guanganmen Hospital

\section{Yu Wei}

China Academy of Chinese Medical Sciences Guanganmen Hospital

\section{Jing-Hui Zheng}

GuangXi Traditional Chinese Medical University: GuangXi University of Chinese Medicine

\section{Lin-Hua Zhao}

China Academy of Chinese Medical Sciences Guanganmen Hospital

\section{Xiaolin Tong ( $\square$ tongxiaolin66@126.com )}

China Academy of Chinese Medical Sciences

\section{Research}

Keywords: diabetic nephropathy, Salvia Miltiorrhiza, molecular mechanism, network pharmacology, molecular docking

Posted Date: October 8th, 2020

DOl: https://doi.org/10.21203/rs.3.rs-85980/v1

License: (c) (1) This work is licensed under a Creative Commons Attribution 4.0 International License. Read Full License

Version of Record: A version of this preprint was published at Bioscience Reports on June 1st, 2021. See the published version at https://doi.org/10.1042/BSR20203520. 


\section{Abstract}

\section{Background}

The mechanisms underlying the therapeutic effect of Salvia Miltiorrhiza (SM) against diabetic nephropathy (DN) using systematic network pharmacology and molecular docking methods were examined.

Methods

TCMSP database was used to screen the active ingredients of SM. Gene targets were obtained using Swiss Target Prediction and TCMSP databases. Related targets of DN were retrieved from the Genecards and DisGeNET databases. Next, a PPI network was constructed using the common targets of SM-DN in the STRING database. The Metascape platform was used for GO function analysis and Cytoscape plug-in ClueGO was used for KEGG pathway enrichment analysis. Molecular docking was performed using iGEMDOCK and AutoDock Vina software. Pymol and LigPlos were used for mapping the network.

Results

Sixty-six active ingredients and 189 targets were screened from SM. Among them, 64 targets overlapped with DN targets. The PPI network diagram revealed that AKT1, VEGFA, IL6, TNF, MAPK1, TP53, EGFR, STAT3, MAPK 14, and JUN were the top 10 relevant targets. GO and KEGG analyses mainly focused on advanced glycation end products, oxidative stress, inflammatory response, and immune regulation. Molecular docking revealed that the potential target genes closely related to DN, including TNF, NOS2, and $A K T 1$, were more stable in combination with salvianolic acid $\mathrm{B}$, and their stability was better than that of tanshinone IIA.

\section{Conclusion}

This study reveals the active components and potential molecular mechanisms involved in the therapeutic effect of SM against DN and provides a reference for the wide application of SM in clinically managing DN.

\section{Background}

Diabetic nephropathy (DN) is a serious complication common in diabetic patients. As the incidence and mortality of patients with diabetes increases year by year, the prevalence of patients with DN rises sharply $\left[{ }^{1}\right]$ and has become one of the leading causes of chronic renal failure $\left.{ }^{2}\right]$. According to reports, DN accounts for approximately $40 \%$ of end-stage kidney disease [ $\left.{ }^{3}\right]$. DN mainly manifests as glomerular hyperfiltration, renal hypertrophy, albuminuria, and even sepsis [ $\left.{ }^{4}\right]$, with persistent proteinuria and gradual 
decline in renal function being its main characteristics [5]. However, its specific molecular mechanism is complicated and unclear, leading to a lack of effective therapies.

In recent years, research on the effects of traditional Chinese medicine (TCM) in regulating blood sugar and lipid metabolism, reducing kidney damage, delaying kidney disease, and preventing glomerular sclerosis and fibrosis have gradually been discovered. Salvia Miltiorrhiza (SM) has a longstanding history as a commonly used TCM for promoting blood circulation in China. Its main functions are reducing blood viscosity, improving hemorheological characteristics, accelerating fibrin degradation $\left.{ }^{6}\right]$, antioxidant activity [ $\left.{ }^{7}\right]$, anti-infection $\left[{ }^{8}\right]$, and improving glucose metabolism disorders $\left[{ }^{9}\right]$. It is often used for microvascular-related diseases such as DN and diabetic retinopathy.

TCM have a multi-target and multi-path intervention strategy that can exert an overall regulatory and synergistic effects, this has certain advantages for DN prevention and individualized treatment. However, the mechanism of SM against DN is unclear.

Network pharmacology is an effective method for studying and clarifying the mechanisms behind drug actions. These methods include chemoinformatics, bioinformatics, network biology, and pharmacology $\left[{ }^{10},{ }^{11}\right]$. The research strategy of network pharmacology is in line with the understanding of disease integrity in TCM $\left[{ }^{12},{ }^{13}\right]$, and provides new ideas and methods for research on TCM [ $\left.{ }^{14}\right]$. In this study, we attempted to utilize network pharmacology technology to explore the main bioactive components of SM, and predict their effective targets and potential mechanisms involved in the treatment of DN at the molecular level. The flowchart is as follows (Fig 1).

\section{Materials And Methods}

\section{Determination and Screening of Active Components of SM}

SM components were retrieved from the Traditional Chinese Medicine System Pharmacology Database [TCMSP, http://tcmspw.com/ tcmsp. Php]. We used pharmacokinetic information retrieval filters for absorption, distribution, metabolism, and excretion (ADME) screening based on oral bioavailability (OB) greater than or equal to $30 \%$ and Drug-likeness (DL) greater than or equal to 0.18 . and active compounds selected without potential target information were excluded. Supplementing the unpredicted active compounds based on published literature reports.

\section{Construction of an Active Component-Target Network}

Obtain targets from the TCMSP and Swiss Target Prediction database (http://www. Swisstargetprediction. ch). Afterwards, the targets were standardized in the UniProt (https://www.uniprot.org) database with the property set to "reviewed" and "human" [15]. After removing duplicates, a database of SM compounds and their targets was constructed. Finally, a visual network was established using Cytoscape v.3.6.0 software. 


\section{Determination of Potential DN-Related Targets}

DN-related targets were retrieved from the Human Gene Database (GeneCards, https: //www.genecards. org/), as well as from the DisGeNET Database (https://www. disgenet.org/home/). The keyword used was "diabetic nephropathy".

\section{Determination of DN-related Targets of the Active Components}

The screened targets of the active components and DN-related proteins were imported into the Venn Diagram (http://bioinformatics.psb.ugent.be/webtools/Venn/) platform for analysis, and the common ones were identified as DN-related targets of the active components for further analysis.

\section{Protein-Protein Interaction (PPI) Network of DN-related Targets of the Active Components}

In order to study the interactions between the active components of SM and their target proteins, the drugdisease intersection target genes were searched using the interaction database platform STRING v.11.0 (https://string-db.org/), and a PPI network was constructed. STRING is a comprehensive multifunctional data platform $\left[{ }^{16},{ }^{17}\right]$ and aims to provide protein-protein interaction evaluation and integration $\left[{ }^{18}\right]$. In our database search, the species was limited to "Homo sapiens", the confidence score cutoff was set at 0.4, and the rest of the settings were default.

\section{Network Construction and Analysis}

The targets of SM among DN-related proteins identified using STRING were further analyzed using the Cytoscape v.3.6.0 software to visualize and analyze the interaction network. Using the network analysis plug-in in the software to count the nodes in the network graph and analyze its role in the graph.

\section{Gene Ontology (GO) Functional Analysis}

The Metascape Platform (http://metascape. Org/gp /index. html) has a comprehensive annotation function that updates the gene annotation data every month [ $\left.{ }^{19}\right]$. The SM regulates DN abnormalities entered into the Metascape platform, set $\mathrm{P}<0.01$, analyzes their main biological processes, and performs enrichment analysis. It saves the data results and uses biological online tools to visualize the data.

\section{Kyoto Encyclopedia of Genes and Genomes (KEGG) Pathway Enrichment Analysis}

KEGG pathway enrichment analysis was conducted using the Cytoscape plug-in ClueGO. The candidate DN-related genes targeted by SM were entered into the ClueGO plug-in, with P set to $<0.01$ and kappa score set to $\geq 0.53$.

\section{Molecular Docking}

Using KEGG pathway enrichment analysis, we identified the potential DN-related genes targeted by SM components. These targets were further confirmed by molecular docking with the experimentally verified 
active components. These components were Tanshinone IIA, which can improve kidney hypertrophy and 24-hour urine protein excretion $\left[{ }^{20}\right]$, and salvianolic acid B, which can inhibit the proliferation of mesangial cells and the production of extracellular matrix induced by high glucose in a dose-dependent manner in SM $\left[{ }^{21}\right]$. The crystal structure of the core genes was obtained from the RCSB Protein Data Bank (PDB, https://www.rcsb.org/) [22]. The compound structure was saved as a docking ligand in MOL2 format. The iGEMDOCK software was used for molecular docking. The software automatically defaults parameters when setting the standard docking.

From the molecular docking results of the iGEMDOCK software, we selected the top 5 receptor proteins with the lowest energy value and the ligand that bound to these receptor proteins most stably and ran AutoDock Vina for docking. Pymol and LigPlos softwares were used for visualization and construction of the network, respectively.

\section{Results}

\section{Screening for Active Components of SM}

Using the TCMSP database to obtain 202 known active compounds in SM, we screened these through the conditions of $\mathrm{OB} \geq 30 \%, \mathrm{DL} \geq 0.18$, and obtained 65 active ingredients that met the conditions.

According to published literature reports, we also selected the known active compound Salvianolic acid B, which was screened out by ADME. Finally, 66 active components were selected. (Table 1).

\section{Determination of Targets of SM Active Components and Active Component-Target Network Construction}

Candidate targets of active components in SM were searched from the databases of TCMSP and Swiss Target Prediction. Then, after uniprot standardization, deduplication occurred. As a result, 189 targets were identified after removal of duplicate data (Table S1). Afterwards, we used cytoscape 3.6.0 software to build a "herbs-ingredients-targets" interaction Network, as shown in Figure 2.

\section{Determination of DN-Related Targets}

The DN-related disease targets obtained from the GeneCards and DisGeNET databases were 1189 and 3084 , respectively.

\section{Drug-Disease Intersection Targets}

Venn analysis was performed on 189 targets of SM components, 1189 and 3084 DN target genes, and 64 drug-disease intersection gene targets were obtained for further analysis, as shown in Figure 3 and Table 2. These targets information were provided in Supplementary Table S2.

\section{PPI Network Analysis}


The drug-disease intersection gene targets (64) were analyzed using the STRING database PPI network, as shown in Figure 4A. From the analysis results, a total of 64 nodes and 704 edges were acquired, and the average node degree was 21.3, with a PPI enrichment p-value of $<1.0 \mathrm{e}-16$ (Fig 4 A). Then, the Import STRING analysis results were analyzed by Cytoscape software. The network analysis plugin was used to count the nodes in the network graph and analyze its role in the graph according to degree. The greater the degree of freedom, the more biological functions the node has in the network. The network was constructed as shown in Figure 4B. Among them, the top ten targets AKT serine/threonine kinase 1 (AKT1), vascular endothelial growth factor A (VEGFA), Interleukin 6 (IL6), Tumor necrosis factor (TNF), Mitogen-activated protein kinase 1 (MAPK1), Tumor protein p53 (TP53), Epidermal growth factor receptor (EGFR), signal transducer and activator of transcription 3 (STAT3), Mitogen-activated protein kinase 14 (MAPK14), and Transcription factor AP-1 (JUN) explain their significance in the network (Fig 4 B).

\section{GO Functional Analysis}

The Metascape data platform was used to enrich and analyze the 64 relevant DN-related targets of SM, and the results were visualized using biological online tools.

A total of 1557 biological processes (BP) were enriched, and the first 20 significantly enriched BP terms were selected for analysis. The results showed that the biological processes involved in SM mainly included cytokine-mediated signaling pathway, apoptotic signaling pathway, positive regulation of cell migration, reactive oxygen species metabolic process, regulation of inflammatory response, regulation of cell-cell adhesion, response to oxygen levels, cellular response to growth factor stimulus, and regulation of protein serine/threonine kinase activity (Fig5 A).

A total of 90 molecular functions (MF) GO terms were enriched, and the first 19 significantly enriched MF terms were selected for analysis based on $P<0.01$. The results showed that the intersection genes were mainly enriched in protein kinase binding, transcription factor binding, cytokine receptor binding, integrin binding, cysteine-type endopeptidase activity involved in apoptotic process, kinase regulator activity, heme binding, protein domain specific binding, endopeptidase activity, nitric-oxide synthase regulator activity, and many other molecular functions related to the above genes (Fig5 B).

A total of 38 cell components (CC) GO terms were enriched, and the first 13 significantly enriched CC terms were selected for analysis according to $P<0.01$. The results showed that the intersection genes were mainly enriched in membrane rafts, RNA polymerase II transcription factor complex, external side of plasma membrane, extracellular matrix, adherens junction, and glutamatergic synapse (Fig $5 \mathrm{C}$ ). Details of the node attribute information of GO analysis results are provided in the supplementary materials (Table S3).

\section{KEGG Pathway Enrichment Analysis}

In order to further reveal the potential mechanism underlying the therapeutic effect of SM against DN, we performed KEGG pathway enrichment analysis on 64 intersection gene targets using the Cytoscape plug- 
in ClueGO. The screen was based on a $p<0.01$ and kappa score $\geq 0.53$, in order to visualize the results of KEGG enrichment(Fig $6 \mathrm{~A}$ ), and we used a pie chart to describe the percentage of genes involved in the different biological functions and signal pathways among the total number of genes that are intersected (Fig 6 B). The results showed that a total of 38 terms were enriched, including the AGE-RAGE signaling pathway in diabetic complications, TNF signaling pathway, JAK-STAT signaling pathway, FoxO signaling pathway, and HIF-1 signaling pathway. In addition, we also found some other pathways, such as Fluid shear stress and atherosclerosis, Platelet activation, Relaxin signaling pathway and so on. These results revealed that SM alleviated DN by improving the organism's immunity, anti-inflammation, relieving advanced glycation end products, antioxidant stress response, and other harmful alien organism-related pathways. Details of node attribute information of the KEGG analysis results are provided in the supplementary materials (Table S4).

\section{Molecular Docking Study}

According to the results of KEGG pathway enrichment analysis, we selected the AGE-RAGE signaling pathway in diabetic complications which had the largest percentage of genes involved in different biological functions and signaling pathways among the total number of intersection genes. According to the drug-target correspondence, the target protein in this pathway is molecularly docked with the corresponding drug components. The 16 target proteins enriched by the AGE-RAGE signaling pathway in diabetic complications are AKT1, BCL2, CASP3, EDN1, ICAM1, IL6, JUN, MAPK1, MAPK14, MMP2, NOS3, NOS2, RELA, STAT3, TNF, and VEGFA. We selected the experimentally verified tanshinone IIA and salvianolic acid $B$ from among the SM active molecules, and molecularly docked with 16 target proteins. The matching energy of small molecules and large molecules determines the degree of binding. If the matching energy is relatively low, it indicates that the conformation of small molecules and large molecules is stable. The 16 potential targets of DN had better binding stability to salvianolic acid $B$ than the tanshinone IIA (Table 3).

Using AutoDock Vina software, the five target proteins (AKT1, NOS2, TNF, JUN, and RELA) with the lowest energy value in the molecular docking by iGEMDOCK software were molecularly docked with Salvianolic acid $B$. Figure 7 shows the best docking combination for the target protein and the active component salvianolic acid B, including TNF, NOS2, and AKT1. These have the best combination with salvianolic acid $B$, and the binding energy is $-9.3 \mathrm{kcal} / \mathrm{mol},-6.6 \mathrm{kcal} / \mathrm{mol}$, and $-6.4 \mathrm{kcal} / \mathrm{mol}$ respectively. This shows that salvianolic acid $B$ has good binding ability with the targets.

\section{Discussion}

TCM mechanisms of action are complicated with multiple components and targets. When the pathogenesis of a disease is not clear yet, it becomes more difficult to analyze the mechanism of action of Chinese medicine. Network pharmacology is a method that combines system network analysis and pharmacology. It can systematically study the effective components, targets, and pathways of drugs at 
the molecular level, so as to understand the interaction between components, targets, and pathways. Therefore, network pharmacology research methods provide new ideas and methods for TCM research.

In this study, the results of the TCM-component-target network analysis showed that luteolin, tanshinone IIA, salviolone, salvianolic acid B, dihydrotanshinlactone, and other active ingredients can act on multiple targets in the network. This finding suggests that these components may be important for the therapeutic effect of SM against DN and warrant further exploration. Luteolin has the most potential targets, followed by Tanshinone IIA. According to reports, luteolin can not only increase insulin-mediated glucose uptake and enhance insulin sensitivity [ $\left.{ }^{23}\right]$, but also inhibit high glucose-induced vascular endothelial growth factor (VEGF) [ ${ }^{24}$, reducing reactive oxygen species (ROS) generation, and reducing lipid accumulation [25] This shows that luteolin can improve insulin resistance and regulate glucose and lipid metabolism. Tanshinone IIA reduces vascular intimal hyperplasia, improves tissue blood perfusion, improves kidney microcirculation, removes intracellular oxygen free radicals, improves blood lipids, promotes anticoagulation and various other functions $\left[{ }^{26}\right]$, such as anti-inflammatory and antioxidant effects $\left[{ }^{27},{ }^{28}\right]$, thereby reducing kidney damage. Salvianolic acid $B$ is a water-soluble compound with the highest activity and highest salvianolic acid content $\left.{ }^{29}\right]$. A number of basic studies have shown that salvianolic acid $B$ has potential therapeutic effects on renal microcirculation. Salvianolic acid B has anti-oxidation $\left[{ }^{30}\right]$, antiinflammatory $\left[{ }^{31}\right]$, neuroprotection $\left[{ }^{32}\right]$, and anti-fibrosis $\left[{ }^{33}\right]$ effects. Current research reports show that salvianolic acid $B$ can be delivered to the kidney to reduce the progression of renal fibrosis $\left[{ }^{34}\right]$, thereby protecting renal function and delaying DN progression.

The PPI network of 64 targets of SM acting on DN showed that AKT1, VEGFA, IL6, TNF, MAPK1, TP53, EGFR, STAT3, MAPK14, and JUN are the top 10 targets in the PPI network according to degree value. These genes are regarded as core genes and may play an important role in the therapeutic effect of SM against DN. These genes are involved in oxidative stress, inflammation, vascular permeability, and immune regulation. For example, the activation of $A K T 1$ has the effect of promoting cell proliferation and inhibiting cell apoptosis. It is an important gene in the immune inflammatory mechanism of DN [ $\left.{ }^{35}\right]$. It is closely related to mesangial matrix proliferation, basement membrane thickening, podocyte damage, and renal tubular epithelial cell transdifferentiation [ $\left.{ }^{36}\right]$; IL 6 and TNF have immunomodulatory and proinflammatory effects $\left[{ }^{37}\right]$; VEGFA is related to the vascular permeability of patients with $\mathrm{DN}\left[{ }^{38}\right]$; the activation of EGFR upregulates the production of ROS and endoplasmic reticulum stress, and the onset of DN plays an important role in this mechanism [ $\left.{ }^{39}\right]$. Therefore, it can be inferred that luteolin, tanshinone IIA, and salvianolic acid $B$, the main components of salvianolic acid $B$, can reduce oxidative stress and inhibit the expression of inflammatory mediators such as IL-10, IL-6, TNF, etc., thus delaying DN progression.

To predict the mechanism underlying the therapeutic effect of SM against DN, we performed $G 0$ enrichment analysis of 64 potential targets. As shown in Figure 5A, the first 20 terms of BP are mainly related to cytokines, apoptosis, reactive oxygen species, and inflammation regulation. For example, cytokine-mediated signaling pathway, apoptosis signaling pathway, reactive oxygen species metabolic 
process, regulation of inflammatory response, etc. Relevant studies have shown that the occurrence and development of DN is related to cell dysfunction and damage $\left[{ }^{40}, 41\right]$, chronic inflammatory infiltration $\left[{ }^{42}\right]$, cell apoptosis, and oxidative stress [ $\left.{ }^{43}\right]$. This indicates that the main target genes are important for multiple BP. MF enrichment analysis mainly included cytokine receptor binding, integrin binding, endopeptidase activity, transcription factor binding, protein kinase binding, and heme binding (Figure 5B). The target genes involved mainly include VEGFA, PTGS2, DDP4, TNF, and NOS2, which mainly focused on oxidative stress, inflammatory response, and immune regulation. Among them, DDP4 inhibitors is of great significance for reducing the blood sugar levels in diabetic patients and delaying the occurrence and development of DN $\left[{ }^{44}, 45\right]$. In addition, as shown in Figure $5 \mathrm{C}$, cellular components mainly include membrane raft, RNA polymerase II transcription factor complex, external side of plasma membrane, extracellular matrix, and adherens junction. These enriched functions also involve top targets, such as TNF and JUN. At the same time, this also illustrates the complexity of the pathological mechanism of DN.

To further explore the potential mechanism of SM in treating DN, we conducted KEGG analysis on 64 potential targets of SM acting on DN. As shown in Figure 6, the pathways related to DN, including the AGE-RAGE signaling pathway in diabetic complications, TNF signaling pathway, JAK-STAT signaling pathway, and FoxO signaling pathway, mainly involve three aspects: (1) accumulation of advanced glycation end products: Normally, the glycation reaction proceeds very slowly. However, the response is obviously accelerated in the hyperglycemic state, and the aggregation of AGEs in tissues and their binding with RAGE, a specific receptor, produces cytotoxic effects and damages the kidneys, which may be the key factors contributing to DN. Most studies have shown that the AGE/RAGE signaling pathway can promote the expression of NF-KB $\left[{ }^{46}\right]$, upregulate TGF-beta 1 , VEGF $\left[{ }^{47}\right]$, and activate NADPH oxidases, etc., cause the expression and release of inflammatory factors and adhesion factors, increase vascular permeability, increase the expression of connective tissue growth factor, and enhance oxidative stress, thus increasing proteinuria, promoting renal fibrosis, leading to the occurrence and development of DN. In addition, other studies have shown that the interaction between AGEs and RAGE leads to vasoconstriction, procoagulant state $\left[{ }^{48}\right]$, accelerates renal vascular aging and injury $\left[{ }^{49}\right]$, and further promotes DN progression. (2) Immune inflammation regulation: TNF has immunomodulatory and proinflammatory effects [37]. TNF-a stimulates the aggregation and adhesion of inflammatory cells, increases the permeability of microvessels, and impairs glomeruli through an inflammatory response $\left.{ }^{50}\right]$. Some studies have confirmed that TNF-a levels are significantly increased in DN patients and positively correlated with the course of disease $\left[{ }^{51},{ }^{52}\right]$. In addition, many studies have shown that activation of the JAK/STAT signaling pathway can cause immune inflammation in renal tissue $\left[{ }^{53}, 54\right]$, and mediates mesangial proliferation and renal tissue fibrosis associated with DN [ $\left.{ }^{55}\right]$. (3) Oxidative stress: FoxO mainly regulates oxidative stress, apoptosis, and immune response through transcription and transmission of various growth factors and cytokine signals, among which FoxO1 plays an important role in the pathogenesis of kidney disease $\left.{ }^{56}\right]$. Fox01 activation can inhibit podocyte epithelialmesenchymal cell transformation induced by high glucose and improve proteinuria and renal damage in diabetic mice $\left[{ }^{57}\right]$. In addition, we found other pathways, such as proteoglycans in cancer, fluid shear 
stress, and atherosclerosis. This indicates that SM has potential applications in tumors, arteriosclerosis, and other diseases. Based on the above multiple pathways, it is speculated that SM may delay the progression of DN and protect renal function by participating in advanced glycation end-products, oxidative stress, inflammatory response, immune regulation, and other processes.

To further explore the potential molecular mechanism of SM in the treatment of DN, we conducted molecular docking studies on 16 target genes closely related to DN screened by KEGG, using the corresponding experimentally validated key components Tanshinone IIA and Salvianolic acid B as ligands. The results showed that 16 potential target genes had good binding with salvianolic acid $B$, and their stability was better than the Tanshinone IIA. The binding energies of these docking results further helped to refine the targets for SM.

\section{Conclusions}

In conclusion, this study analyzed the mechanisms underlying the therapeutic effect of SM against DN using network pharmacology and analysis of a PPI network showing the interactions between SM active components and targeted DN-related proteins and determined the synergistic effects between the herbs in SM. Our findings revealed that SM exerted its pharmacological effects against DN through "multi components-multi targets-multi pathways" that were mainly involved in advanced glycation end-products, oxidative stress, inflammatory response, and immune regulation. Further, our findings offer a reference for further investigation of the mechanism underlying the therapeutic effect of SM against DN. This study also had some limitations. We only explored the effect of SM on DN at the level of network pharmacology. However, the current network information technology is not comprehensive, and the accuracy of database data and real-time updates needs to be improved. Therefore, the results obtained from this analysis need further verification with respect to the corresponding pharmacodynamics, and mechanistic experiments are needed to explain the complex multi-target, multi-pathway, and synergistic interactions involved in the therapeutic effects of TCM.

\section{Abbreviations}

TCM: Traditional Chinese medicine; TCMSP: Traditional Chinese Medicine Systems Pharmacology; OB: Oral bioavailability; DL: Drug likeness; PPI: Protein-protein interaction; STRING: Search Tool for the Retrieval of Interacting Genes/Proteins database; GO: Gene Ontology; BP: Biological processes; MF: Molecular functions; CC: Cellular components; KEGG: Kyoto Encyclopedia of Genes and Genomes; PDB: Protein Data Bank; SM :Salvia Miltiorrhiza; DN: Diabetic Nephropathy.

\section{Declarations}

Acknowledgements

Not applicable. 
Authors' Contributions

X-LT, L-HZ, and L-LZ conceived and designed the research methods. L-LZ, L-H, X-MW, Y-W, and J-HZ collected the data. $L-L Z$ and $L-H$ analysed the data. $L-L Z, L-H$, and $X-M W$ wrote the original draft. X-LT and $\mathrm{L}-\mathrm{HZ}$ reviewed and edited the manuscript. All authors read and approved the final manuscript.

\section{Funding}

This study was supported by the National Natural Science Foundation of China (grant number 81430097) and the Major Achievement Guidance Project of Traditional Chinese Medicine Science and Technology (grant number 44223).

\section{Availability of data and materials}

The datasets used and/or analysed during the current study are available from the corresponding author on reasonable request.

\section{Ethics approval and consent to participate}

Not applicable.

\section{Consent for publication}

Not applicable.

\section{Competing interests}

The authors declare no conflict of interest.

\section{References}

1. Reutens AT, Atkins RC. Epidemiology of Diabetic Nephropathy. Contributions to. nephrology. 2011; 170: $1-7$.

2. Gembillo G, Cernaro V, Salvo A, Siligato R, Santoro D. Role of Vitamin D Status in Diabetic Patients with Renal Disease. Medicina (Kaunas, Lithuania). 2019; 55(6): 273-279.

3 . Keri KC, Samji NS, Samuel B. Diabetic Nephropathy: Newer Therapeutic Perspectives. Journal of Community Hospital Internal Medicine Perspectives. 2018; 8(4):200-207.

4. Yang H, Kan QE, Su Y, Man H. Long non-coding RNA CASC2 improves diabetic nephropathy by inhibiting JNK pathway. Experimental \& Clinical Endocrinology \& Diabetes. 2019, 127(8):533-537.

5. Wang J, Li H, Tan M, Gao G, Zhang Y, Ding B, Su X, Kong XC, Ma JH. Association between Thyroid Function and Diabetic Nephropathy in Euthyroid Subjects with Type 2 Diabetes Mellitus: A CrossSectional Study in China. Oncotarget. 2019;102(2):88-97. 
6 . Wu LC, Lin X, Sun H. Tanshinone $\triangle A$ Protects Rabbits against LPS-Induced Disseminated Intravascular Coagulation (Dic). Acta Pharmacologica Sinica. 2012;33(10):1254-1259.

7. Nickavar B, Rezaee J, Nickavar A. Iranian Effect-Directed Analysis for the Antioxidant Compound in Salvia erticillata. Iran J Pharm Res. 2016;15 (1) :241-246.

8. Liu X, Meng J. Tanshinone $\triangle A$ Ameliorates Lipopolysaccharide-Induced Inflammatory. Response in Bronchial Epithelium Cell Line BEAS-2B by Down-Regulating MiR-27a. Biomedicine \& pharmacotherapy.2018;104:158-164.

9. Kim EJ, Jung SN, Son KH, Kim SR, Ha TY, Park MG, Jo IG, Park JG, Choe WC, Kim S, Ha J. Antidiabetes and Antiobesity Effect of Cryptotanshinone Via Activation of Amp-Activated Protein Kinase. Molecular pharmacology. 2007;72(1):62-72.

10. Zhang JY, Liang RX, Wang L, Yang B. Effects and Mechanisms of Danshen-Shanzha Herb-Pair for Atherosclerosis Treatment Using Network Pharmacology and Experimental Pharmacology. Journal of Ethnopharmacology. 2019; 229:104-114.

11. Chen LD, Cao Y, Zhang H, Lv DY, Zhao YH, Liu YJ, Ye G, Chai YF. Network. Pharmacology-Based Strategy for Predicting Active Ingredients and Potential Targets of Yang Xin Shi Tablet for Treating Heart Failure. Journal of ethnopharmacology. 2018; 219:359-368.

12. Xu J, Wang F, Guo JJ, Xu CS, Cao YZ, Fang ZL, Wang QW. Pharmacological Mechanisms Underlying. the Neuroprotective Effects of Alpinia Oxyphylla Miq. On Alzheimer's Disease. International journal of molecular sciences. 2020; 21(6):2071-2085.

13. Ding ZH, Zhong RX, Yang YN, Xia TY, Wang WJ, Wang Y, Xing N, Luo Y, Li SY, Shang LF. Systems Pharmacology Reveals the Mechanism of Activity of Ge-Gen-Qin-Lian Decoction against LPS-Induced Acute Lung Injury: A Novel Strategy for Exploring Active Components and Effective Mechanism of TCM Formulae. Pharmacological Research. 2020; 156:1047-1059.

14. Zhang YQ, Mao X, Guo QY, Lin N, Li S. Network Pharmacology-Based Approaches Capture Essence of Chinese Herbal Medicines. Chinese Herbal Medicines. 2016;8(2):107-116.

15. Consortium TU. Reorganizing the Protein Space at the Universal Protein Resource (Uniprot). Nucleic Acids Research.2012;40(D1):71-75.

16. Damian S, Annika LG, David L, Alexander J, Wyder S, Jaime HC, Milan S, Nadezh TD, John HM, Peer B, Jensen LJ, Mering CV. String V11: Protein-Protein Association Networks with Increased Coverage, Supporting Functional Discovery in Genome-Wide Experimental Datasets. Nucleic acids research. 2019; 47(D1):607-613.

17. Huang C, Li R, Shi W, Huang Z. Discovery of the Anti-Tumor Mechanism of. Calycosin against. Colorectal Cancer by Using System Pharmacology Approach. Med Sci Monit. 2019; 25:5589-5593. 
18. Damian S, Andrea F, Stefan W, Kristoffer F, Davide H, Jaime H C, Milan S, Alexander R, Alberto S, Tsafou KP. String V10: Protein-Protein Interaction Networks, Integrated over the Tree of Life. Nucleic Acids Research. 2015;43(D1):447-452.

19. Zhou YY, Zhou B, Pache L, Chang M, Khodabakhshi AH, Tanaseichuk O, Benner C, Chanda SK. Metascape Provides a Biologist-Oriented Resource for the Analysis of Systems-Level Datasets. Nature Communications. 2019; 10(1):1523-1533.

20. Kim SK, Jung KH, Lee BC. Protective Effect of Tanshinone $\mathbb{A}$ A on the Early Stage of Experimental Diabetic Nephropathy. Biological \& Pharmaceutical Bulletin. 2009; 32(2):220-224.

21. Luo P, Tan ZH, Zhang ZF, Li HH, Mo Z. Inhibitory Effects of Salvianolic Acid B on the High. GlucoseInduced Mesangial Proliferation Via NF-kappaB-dependent Pathway. Biological \& Pharmaceutical Bulletin. 2008;31(7):1381-1386.

22 . Berman H, Henrick K, Nakamura H. Announcing the Worldwide Protein Data Bank. Nature. Structural Biology. 2003;10(12):980.

23 . Ding L, Jin DZ, Chen XL. Luteolin Enhances Insulin Sensitivity Via Activation of Ppary Transcriptional Activity in Adipocytes. Journal of Nutritional Biochemistry. 2010; 21(10):941-947.

24. Lu HE, Chen Y, Sun XB, Tong B, Fan XH. Effects of Luteolin on Retinal Oxidative Stress and Inflammation in Diabetes. Rsc Advances. 2015;5(7):4898-4904.

25. Liu JF, Ma Y, Wang Y, Du ZY, Shen JK, Peng HL. Reduction of Lipid. Accumulation in Hepg2 Cells by Luteolin is Associated with Activation of AMPK and Mitigation of Oxidative Stress. Phytotherapy Research. 2011; 25(4):588-596.

26 . Amenedo M, Residential R. EGFR Mutations and ALK Alterations in Never-smoking Lung. Cancer Cases. European Respiratory Journal. 2016,48(5):1462-1470.

27. Gao HW, Liu X, Sun W, Kang NX, Liu YL, Yang SL, Xu QM, Wang CM, Chen XP. Total Tanshinones Exhibits Anti-Inflammatory Effects through Blocking TLR4 Dimerization Via the MyD88 Pathway. Cell Death \& Disease. 2017;8(8): e3004.

28. Chen X, Wu R, Kong YW, Yang YT, Gao Y, Sun DD, Liu QZ, Dai DJ, Lu ZY, Wang NS, Ge S, Wang F. Tanshinone IIA Attenuates Renal Damage in STZ-Induced Diabetic Rats Via Inhibiting Oxidative Stress and Inflammation. Oncotarget. 2017;8(19):31915-31922.

29. Zhai JH, Tao L, Zhang YM, Gao H, Qu XY, Song YQ, Zhang SX. Salvianolic Acid B Attenuates Apoptosis of HUVEC Cells Treated with High Glucose or High Fat Via Sirt1 Activation. Evidence-Based Complementary and Alternative Medicine. 2019;2019(21):1-11. 
30. Zhang YF, Xu LW, Liang K, Zhou LH, Ge YZ, Jia RP. Protective Effect of. Salvianolic Acid B against Oxidative Injury Associated with Cystine Stone Formation. Urolithiasis. 2019; 47(6):503-510.

31. Liu QM, Shi XG, Tang LY, Xu WH, Jiang S, Ding WF, Feng Q, Chu HY, Ma YY, Li Y, Lu JY, Pu WL, Zhou XD, Li J, Wang JC, Wu WY. Salvianolic Acid B Attenuates Experimental Pulmonary Inflammation by Protecting Endothelial Cells against Oxidative Stress Injury. European Journal of Pharmacology. 2018; 840: 9-19.

32. Wang QQ, Zhai C, Wahafu A, Zhu YT, Liu YH, Sun LQ. Salvianolic AcidB Inhibits. the Development of Diabetic Peripheral Neuropathy by Suppressing Autophagy and Apoptosis. The Journal of pharmacy and pharmacology. 2019;71(3):417-428.

33. Liu QM, Lu JY, Lin JR, Tang YL, Pu WL, Shi XG, Jiang S, Liu J, Ma YY, Li Y, Xu JH, Li J, Wang JC, Wu WY. Salvianolic Acid B Attenuates Experimental Skin Fibrosis of Systemic Sclerosis. Biomedicine \& Pharmacotherapy. 2019;110: 546-553.

34 . Li J, Zhang CT, He WM, Qiao HZ, Chen JH, Wang KK, Oupick D, Sun M. Coordination-Driven Assembly of Catechol-Modified Chitosan for the Kidney-Specific Delivery of Salvianolic Acid B to Treat Renal Fibrosis. Biomater Sci. 2017;6(1):179-188.

35. Patel RK, Mohan C. PI3K/AKT Signaling and Systemic Autoimmunity. Immunologic research. 2005; 31(1):47-55.

36. Ribback S, Cigliano A, Kroeger N, Pilo MG, Dombrowski F. PI3K/AKT/MTOR Pathway Plays a Major Pathogenetic Role in Glycogen Accumulation and Tumor Development in Renal Distal Tubules of Rats and Men. Oncotarget. 2015;6(15):13036-13048.

37. Yi HY, Zhang Y, Yang XF, Li MY, Hu HF, Xiong J, Wang N, Jin JY, Zhang YS, Song Y, Wang X, Chen LH, Lian JQ. Hepatitis B Core Antigen Impairs the Polarization While Promoting the Production of Inflammatory Cytokines of M2 Macrophages Via the TLR2 Pathway. Frontiers in Immunology. 2020;11: 535-549.

38. Onions K, Gamez M, Buckner N, Baker S, Betteridge K, Desideri S, Dallyn B, Ramnath R, Neal C, Farmer L, Mathieson P, Gnudi L, Alitalo K, Bates D, Salmon A, Welsh G, Satchell S, Foster R. VEGFC Reduces Glomerular Albumin Permeability and Protects against Alterations in VEGF Receptor Expression in Diabetic Nephropathy. Diabetes. 2019;68 (1):172-187.

39. Zheng X, Zhao YJ, Zhong P, Wang JY, Weng QY, Qian YY, Han JB, Zou CP, Liang G. EGFR Inhibition Attenuates Diabetic Nephropathy through Decreasing ROS and Endoplasmic Reticulum Stress. Oncotarget. 2017;8(20):32655-32667.

40 . Lu P, Ji X, Wan J, Xu H. Activity of Group 2 Innate Lymphoid Cells Is Associated with Chronic. Inflammation and Dysregulated Metabolic Homoeostasis in Type 2 Diabetic Nephropathy. Scandinavian 
Journal of Immunology. 2017;87(2):99-107.

41 . Tesch GH. Diabetic nephropathy-is this an immune disorder?. Clin Sci (Lond). 2017, 131 (16): 21832199.

42. Fathy SA, Mohamed MR, Ali MM, El-Helaly AE, Alattar AT. Influence of IL-6, IL-10, IFN-y and TNF-a Genetic Variants on Susceptibility to Diabetic Kidney Disease in Type 2 Diabetes Mellitus Patients. Biomarkers. 2019,24(1):43-55.

43. Wu N, Shen H, Liu H, Wang Y, Bai Y, Han P. Acute blood glucose fluctuation. enhances rat aorta endothelial cell apoptosis, oxidative stress and pro-inflammatory cytokine expression in vivo. Cardiovasc Diabetol. 2016;15(1):109-121.

44 . Lamont BJ, Drucker DJ. Differential Antidiabetic Efficacy of Incretin Agonists. Versus Dpp-4 Inhibition in High Fat Fed Mice. Diabetes. 2008;57(1):190-198.

45. Moritoh Y, Takeuchi K, Asakawa T, Kataoka O, Odaka H. Chronic Administration of. Alogliptin, a Novel, Potent, and Highly Selective Dipeptidyl Peptidase-4 Inhibitor, Improves Glycemic Control and Beta-Cell Function in Obese Diabetic Ob/Ob Mice. European Journal of Pharmacology. 2008;588(2-3):325-332.

46. Tobon-Velasco JC, Cuevas E, Torres-Ramos MA. Receptor for Ages (RAGE) as Mediator of NF-kB Pathway Activation in Neuroinflammation and Oxidative Stress. CNS \& Neurological Disorders-Drug Targets. 2014; 13(9):1615-1626.

47. Pang RY, Guan MP, Zheng ZJ, Xue YM. Effects of Metformin on Apoptosis. Induced by Advanced. Glycation End-Products and Expressions of Caspase-3, Bax and Bcl-2 in Human Dermal Fibroblasts in Vitro. Journal of Southern Medical University. 2015;35(6):898-902.

48. Liu FX, Fu YC, Wei CJ, Chen YR,Ma SH, Xu WC. The Expression of GPR109A, NF-kB and II-1 $\beta$ in Peripheral Blood Leukocytes from Patients with Type 2 Diabetes. Ann Clin Lab Sci. 2014;44(4):443-448.

49. Yamagishi SI, Matsui T. Role of Ligands of Receptor for Advanced Glycation End Products (RAGE) in Peripheral Artery Disease. Rejuvenation Research. 2018; 21:456-463.

50 . Rakitianskaia IA, Riabov SI, Azanchevskaia SV, Riabova TS, Popova VB. Role of Intrarenal. Product TNF-Alpha in the Development of Glomerular and Tubulointerstitial Tissues Changes in Elderly Patients with Diabetic Nephropathy. adv gerontol. 2013; 26(4):658-665.

51. Moresco RN, Sangoi MB, Carvalho JM, Tatsch E, Bochi GV. Diabetic Nephropathy: Traditional to Proteomic Markers. Clinica chimica acta. 2013; 421: 17-30.

52. Wu CC, Chen JS, Lu KC, Chen CC, Lin SH, Chu P, Sytwu HK, Lin YF. Aberrant. Cytokines/Chemokines Production Correlate with Proteinuria in Patients with Overt Diabetic Nephropathy. Clinica Chimica Acta. 2010; 411(9-10):700-704. 
53. Liu QJ, Xing LL, Wang L, Yao F, Liu SX, Hao J, Liu W, Duan HJ. Therapeutic. Effects of. Suppressors of Cytokine Signaling in Diabetic Nephropathy. J Histochem Cytochem. 2014;62(2):119-128.

54 . Debbie W, Hani A M, Daniel L, Ariel T, Leonid K, Ralph R, Yael S. Impaired Renal Growth Hormone JAK/STAT5 Signaling in Chronic Kidney Disease. Nephrol Dial Transplant. 2014,29(4):791-799.

55. Zhou YH, Lv C, Wu C, Chen FQ, Shao Y, Wang QY. Suppressor of Cytokine. Signaling (Socs) 2 Attenuates Renal Lesions in Rats with Diabetic Nephropathy. Acta Histochemica. 2014; 116(5): 981-988.

56. Shi WX, Li N, Sui MS. Research Progress of Foxo in Kidney Diseases. Journal of Chinese Practical Diagnosis and Therapy.2018;32(2):205-208.

57. Du MM, Wang QZ, Li W, Ma XJ, Wu L, Guo F, Zhao SY, Huang FJ, Wang HH, Qin GJ. Overexpression of Foxo1 Ameliorates the Podocyte Epithelial-Mesenchymal Transition Induced by High Glucose In vitro and In vivo. Biochemical \& Biophysical Research Communications. 2016;471(4):416-422.

\section{Tables}

Table 1. Basic information on the main active ingredients of SM 


\begin{tabular}{|c|c|c|c|}
\hline Mol ID & Molecule Name & $\mathrm{OB} \%$ & DL \\
\hline MOL001601 & 1,2,5,6-tetrahydrotanshinone & 38.75 & 0.36 \\
\hline MOL001659 & Poriferasterol & 43.83 & 0.76 \\
\hline MOL001771 & poriferast-5-en-3beta-ol & 36.91 & 0.75 \\
\hline MOL001942 & isoimperatorin & 45.46 & 0.23 \\
\hline MOL002222 & sugiol & 36.11 & 0.28 \\
\hline MOL002651 & Dehydrotanshinone II A & 43.76 & 0.4 \\
\hline MOL002776 & Baicalin & 40.12 & 0.75 \\
\hline MOL000569 & digallate & 61.85 & 0.26 \\
\hline MOL000006 & luteolin & 36.16 & 0.25 \\
\hline MOL007036 & $\begin{array}{l}\text { 5,6-dihydroxy-7-isopropyl-1,1-dimethyl-2,3-dihydrophenanthren-4- } \\
\text { one }\end{array}$ & 33.77 & 0.29 \\
\hline MOL007041 & 2-isopropyl-8-methylphenanthrene-3,4-dione & 40.86 & 0.23 \\
\hline MOL007045 & 3a-hydroxytanshinone『a & 44.93 & 0.44 \\
\hline MOL007048 & $\begin{array}{l}\text { (E)-3-[2-(3,4-dihydroxyphenyl)-7-hydroxy-benzofuran-4-yl]acrylic } \\
\text { acid }\end{array}$ & 48.24 & 0.31 \\
\hline MOL007049 & 4-methylenemiltirone & 34.35 & 0.23 \\
\hline MOL007050 & $\begin{array}{l}\text { 2-(4-hydroxy-3-methoxyphenyl)-5-(3-hydroxypropyl)-7-methoxy-3- } \\
\text { benzofurancarboxaldehyde }\end{array}$ & 62.78 & 0.4 \\
\hline MOL007058 & formyltanshinone & 73.44 & 0.42 \\
\hline MOL007059 & 3-beta-Hydroxymethyllenetanshiquinone & 32.16 & 0.41 \\
\hline MOL007061 & Methylenetanshinquinone & 37.07 & 0.36 \\
\hline MOL007063 & przewalskin a & 37.11 & 0.65 \\
\hline MOL007064 & przewalskin b & 110.32 & 0.44 \\
\hline MOL007068 & Przewaquinone B & 62.24 & 0.41 \\
\hline MOL007069 & przewaquinone $\mathrm{c}$ & 55.74 & 0.4 \\
\hline MOL007070 & $\begin{array}{l}\text { (6S,7R)-6,7-dihydroxy-1,6-dimethyl-8,9-dihydro-7H-naphtho[8,7- } \\
\text { g]benzofuran-10,11-dione }\end{array}$ & 41.31 & 0.45 \\
\hline MOL007071 & przewaquinone $f$ & 40.31 & 0.46 \\
\hline MOL007077 & sclareol & 43.67 & 0.21 \\
\hline MOL007079 & tanshinaldehyde & 52.47 & 0.45 \\
\hline
\end{tabular}




\begin{tabular}{|c|c|c|c|}
\hline MOL007081 & Danshenol B & 57.95 & 0.56 \\
\hline MOL007082 & Danshenol A & 56.97 & 0.52 \\
\hline MOL007085 & Salvilenone & 30.38 & 0.38 \\
\hline MOL007088 & cryptotanshinone & 52.34 & 0.4 \\
\hline MOL007093 & dan-shexinkum d & 38.88 & 0.55 \\
\hline MOL007094 & danshenspiroketallactone & 50.43 & 0.31 \\
\hline MOL007098 & deoxyneocryptotanshinone & 49.4 & 0.29 \\
\hline MOL007100 & dihydrotanshinlactone & 38.68 & 0.32 \\
\hline MOL007101 & dihydrotanshinone $\mathbb{Z}$ & 45.04 & 0.36 \\
\hline MOL007105 & epidanshenspiroketallactone & 68.27 & 0.31 \\
\hline MOL007107 & C09092 & 36.07 & 0.25 \\
\hline MOL007108 & isocryptotanshi-none & 54.98 & 0.39 \\
\hline MOL007111 & Isotanshinone II & 49.92 & 0.4 \\
\hline MOL007115 & manool & 45.04 & 0.2 \\
\hline MOL007119 & 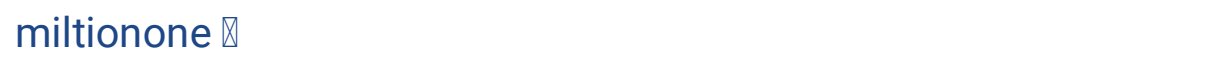 & 49.68 & 0.32 \\
\hline MOL007120 & miltionone $\mathbb{Q}$ & 71.03 & 0.44 \\
\hline MOL007121 & miltipolone & 36.56 & 0.37 \\
\hline MOL007122 & Miltirone & 38.76 & 0.25 \\
\hline MOL007124 & neocryptotanshinone ii & 39.46 & 0.23 \\
\hline MOL007125 & neocryptotanshinone & 52.49 & 0.32 \\
\hline MOL007127 & 1-methyl-8,9-dihydro-7H-naphtho[5,6-g]benzofuran-6,10,11-trione & 34.72 & 0.37 \\
\hline MOL007130 & prolithospermic acid & 64.37 & 0.31 \\
\hline MOL007132 & $\begin{array}{l}\text { (2R)-3-(3,4-dihydroxyphenyl)-2-[(Z)-3-(3,4- } \\
\text { dihydroxyphenyl)acryloyl] oxy-propionic acid }\end{array}$ & 109.38 & 0.35 \\
\hline MOL007141 & salvianolic acid g & 45.56 & 0.61 \\
\hline MOL007142 & salvianolic acid j & 43.38 & 0.72 \\
\hline MOL007143 & salvilenone $\mathbb{Q}$ & 32.43 & 0.23 \\
\hline MOL007145 & salviolone & 31.72 & 0.24 \\
\hline MOL007150 & $\begin{array}{l}\text { (6S)-6-hydroxy-1-methyl-6-methylol-8,9-dihydro-7H-naphtho[8,7- } \\
\text { g]benzofuran-10,11-quinone }\end{array}$ & 75.39 & 0.46 \\
\hline
\end{tabular}




\begin{tabular}{|llrc|}
\hline MOL007151 & Tanshindiol B & 42.67 & 0.45 \\
\hline MOL007152 & Przewaquinone E & 42.85 & 0.45 \\
\hline MOL007154 & tanshinone iia & 49.89 & 0.4 \\
\hline MOL007156 & $\begin{array}{l}\text { (6S)-6-(hydroxymethyl)-1,6-dimethyl-8,9-dihydro-7H-naphtho[8,7- } \\
\text { g]benzofuran-10,11-dione }\end{array}$ & 65.26 & 0.45 \\
\hline MOL006824 & a-amyrin & 45.64 & 0.3 \\
\hline MOL007118 & microstegiol & 39.51 & 0.76 \\
\hline MOL007123 & miltirone \ & 39.61 & 0.28 \\
\hline MOL007149 & NSC 122421 & 44.95 & 0.24 \\
\hline MOL007140 & $\begin{array}{l}\text { (Z)-3-[2-[(E)-2-(3,4-dihydroxyphenyl)vinyl]-3,4-dihydroxy- } \\
\text { phenyl]acrylic acid }\end{array}$ & 34.49 & 0.28 \\
\hline MOL007051 & 6-o-syringyl-8-o-acetyl shanzhiside methyl ester & 88.54 & 0.26 \\
\hline MOL007074 & salvianolic acid b & 46.69 & 0.71 \\
\hline
\end{tabular}

Table 2. Potential targets of SM against DN 


\begin{tabular}{|c|c|c|c|}
\hline $\begin{array}{l}\text { Serial } \\
\text { number }\end{array}$ & Protein name & $\begin{array}{l}\text { Gene } \\
\text { name }\end{array}$ & $\begin{array}{l}\text { UniProt } \\
\text { ID }\end{array}$ \\
\hline 1 & peroxisome proliferator activated receptor gamma & PPARG & P37231 \\
\hline 2 & vascular endothelial growth factor $A$ & VEGFA & P15692 \\
\hline 3 & insulin receptor & INSR & P06213 \\
\hline 4 & interleukin 6 & IL6 & P05231 \\
\hline 5 & nitric oxide synthase 3 & NOS3 & P29474 \\
\hline 6 & tumor necrosis factor & TNF & P01375 \\
\hline 7 & solute carrier family 2 member 4 & SLC2A4 & P14672 \\
\hline 8 & AKT serine/threonine kinase 1 & AKT1 & P31749 \\
\hline 9 & signal transducer and activator of transcription 3 & STAT3 & P40763 \\
\hline 10 & dipeptidyl peptidase 4 & DPP4 & P27487 \\
\hline 11 & intercellular adhesion molecule 1 & ICAM1 & P05362 \\
\hline 12 & tumor protein p53 & TP53 & P04637 \\
\hline 13 & interleukin 10 & IL10 & P22301 \\
\hline 14 & endothelin 1 & EDN1 & P05305 \\
\hline 15 & CD40 ligand & CD40LG & P29965 \\
\hline 16 & matrix metallopeptidase 9 & MMP9 & P14780 \\
\hline 17 & interleukin 4 & IL4 & P05112 \\
\hline 18 & nitric oxide synthase 2 & NOS2 & P35228 \\
\hline 19 & interferon gamma & IFNG & P01579 \\
\hline 20 & interleukin 2 & IL2 & P60568 \\
\hline 21 & heme oxygenase 1 & HMOX1 & P09601 \\
\hline 22 & matrix metallopeptidase 2 & MMP2 & P08253 \\
\hline 23 & mitogen-activated protein kinase 1 & MAPK1 & P28482 \\
\hline 24 & prostaglandin-endoperoxide synthase 2 & PTGS2 & P35354 \\
\hline 25 & mitogen-activated protein kinase 14 & MAPK14 & Q16539 \\
\hline 26 & nuclear receptor subfamily 3 group C member 2 & NR3C2 & P08235 \\
\hline 27 & caspase 3 & CASP3 & P42574 \\
\hline 28 & Jun proto-oncogene, AP-1 transcription factor subunit & JUN & P05412 \\
\hline
\end{tabular}




\begin{tabular}{|c|c|c|c|}
\hline 29 & xanthine dehydrogenase & $\mathrm{XDH}$ & P47989 \\
\hline 30 & estrogen receptor 1 & ESR1 & P03372 \\
\hline 31 & matrix metallopeptidase 1 & MMP1 & P03956 \\
\hline 32 & $\begin{array}{l}\text { phosphatidylinositol-4,5-bisphosphate 3-kinase catalytic } \\
\text { subunit gamma }\end{array}$ & PIK3CG & P48736 \\
\hline 33 & endothelin receptor type $A$ & EDNRA & P25101 \\
\hline 34 & epidermal growth factor receptor & EGFR & P00533 \\
\hline 35 & serine protease 1 & PRSS1 & P07477 \\
\hline 36 & RELA proto-oncogene, NF-kB subunit & RELA & Q04206 \\
\hline 37 & caspase 9 & CASP9 & P55211 \\
\hline 38 & prostaglandin-endoperoxide synthase 1 & PTGS1 & P23219 \\
\hline 39 & 5-hydroxytryptamine receptor $2 \mathrm{~A}$ & HTR2A & P28223 \\
\hline 40 & fatty acid synthase & FASN & P49327 \\
\hline 41 & cyclin dependent kinase inhibitor $1 \mathrm{~A}$ & CDKN1A & P38936 \\
\hline 42 & coagulation factor $X$ & F10 & P00742 \\
\hline 43 & glutathione S-transferase pi 1 & GSTP1 & P09211 \\
\hline 44 & BCL2 apoptosis regulator & BCL2 & P10415 \\
\hline 45 & integrin subunit beta 3 & ITGB3 & P05106 \\
\hline 46 & protein tyrosine phosphatase non-receptor type 2 & PTPN2 & P17706 \\
\hline 47 & MDM2 proto-oncogene & MDM2 & Q00987 \\
\hline 48 & integrin subunit alpha $2 b$ & ITGA2B & P08514 \\
\hline 49 & matrix metallopeptidase 12 & MMP12 & P39900 \\
\hline 50 & nuclear receptor subfamily 1 group I member 2 & NR1/2 & 075469 \\
\hline 51 & caspase 7 & CASP7 & P55210 \\
\hline 52 & Lymphocyte differentiation antigen CD38 & CD38 & P28907 \\
\hline 53 & Glyoxalase I & GL01 & Q04760 \\
\hline 54 & Arachidonate 12-lipoxygenase & ALOX12 & P18054 \\
\hline 55 & Aldose reductase (by homology) & AKR1B1 & P15121 \\
\hline 56 & Protein-tyrosine phosphatase $1 \mathrm{C}$ & PTPN6 & P29350 \\
\hline 57 & LXR-alpha & NR1H3 & Q13133 \\
\hline
\end{tabular}




\begin{tabular}{|llll|}
\hline 58 & Protein-tyrosine phosphatase 2C & PTPN11 & Q06124 \\
\hline 59 & Arginase-1(by homology) & ARG1 & P05089 \\
\hline 60 & Poly [ADP-ribose] polymerase-1 & PARP1 & P09874 \\
\hline 61 & Adenosine A1 receptor (by homology) & ADORA1 & P30542 \\
\hline 62 & NADPH oxidase 4 & NOX4 & Q9NPH5 \\
\hline 63 & Tyrosine-protein kinase SYK & SYK & P43405 \\
\hline 64 & Cytochrome P450 19A1 & CYP19A1 & P11511 \\
\hline
\end{tabular}

Table 3. Docking of Tanshinone IIA and Salvianolic Acid B with target protein molecules $\left(\mathrm{kcal} \cdot \mathrm{mol}^{-1}\right)$ 


\begin{tabular}{|lllll|}
\hline Target name & PDBID & Tanshinone IIA & Salvianolic acid B & Canagliflozin \\
\hline MMP2 & 1EAK & -88.21 & -127.25 & -109.23 \\
\hline EDN1 & 1EDP & -62 & -110.92 & -92.83 \\
\hline RELA & 1NFI & -81.98 & -151.32 & -92.46 \\
\hline NOS3 & 1NIW & -92.71 & -131.05 & -113.3 \\
\hline JUN & 1S9K & -87.31 & -147.78 & -99.95 \\
\hline AKT1 & 1UNQ & -79.22 & -148.03 & -102.74 \\
\hline BCL2 & 1YSW & -78.34 & -129.71 & -113.85 \\
\hline TNF & 2E7A & -90.62 & -146.4 & -113.05 \\
\hline MAPK14 & 2NPQ & -84.34 & -139.24 & -102.14 \\
\hline BCL2 & 2O2F & -82.16 & -133.24 & -99.02 \\
\hline BCL2 & 2O21 & -89.69 & -136.58 & -105.02 \\
\hline BCL2 & 2O22 & -97.25 & -135.9 & -104.91 \\
\hline NOS2 & 3E7G & -89.58 & -162.49 & -109.28 \\
\hline CASP3 & 3KJF & -81.44 & -130.26 & -91.52 \\
\hline VEGFA & 3V2A & -76.85 & -121.29 & -91.19 \\
\hline IL6 & 4CNI & -89.55 & -125.52 & -98.18 \\
\hline MAPK1 & 4IZ5 & -78.5 & -144.95 & -97.71 \\
\hline STAT3 & 4ZIA & -84.1 & -136.45 & -103.68 \\
\hline ICAM1 & 5MZA & -80.39 & -125.56 & -98.36 \\
\hline
\end{tabular}

Figures 

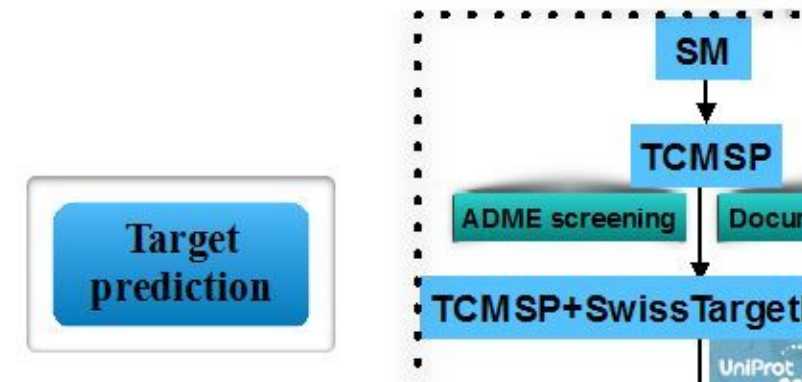

ADME screening Document Retrieval

TCMSP+Swiss TargetPrediction
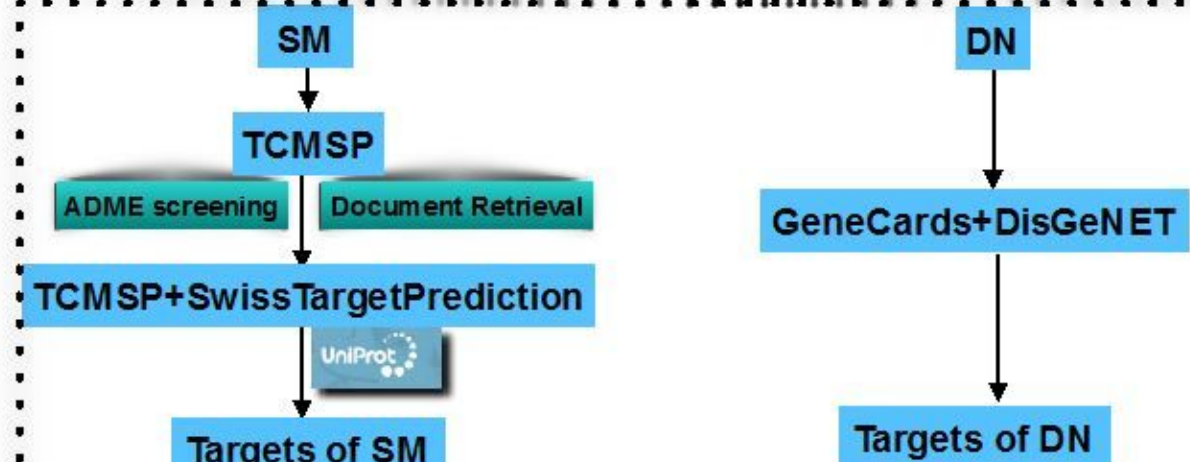

Network construction

Functional enrichment

analysis

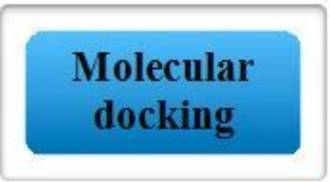

Figure 1

Flowchart based on network pharmacology and molecular docking. 

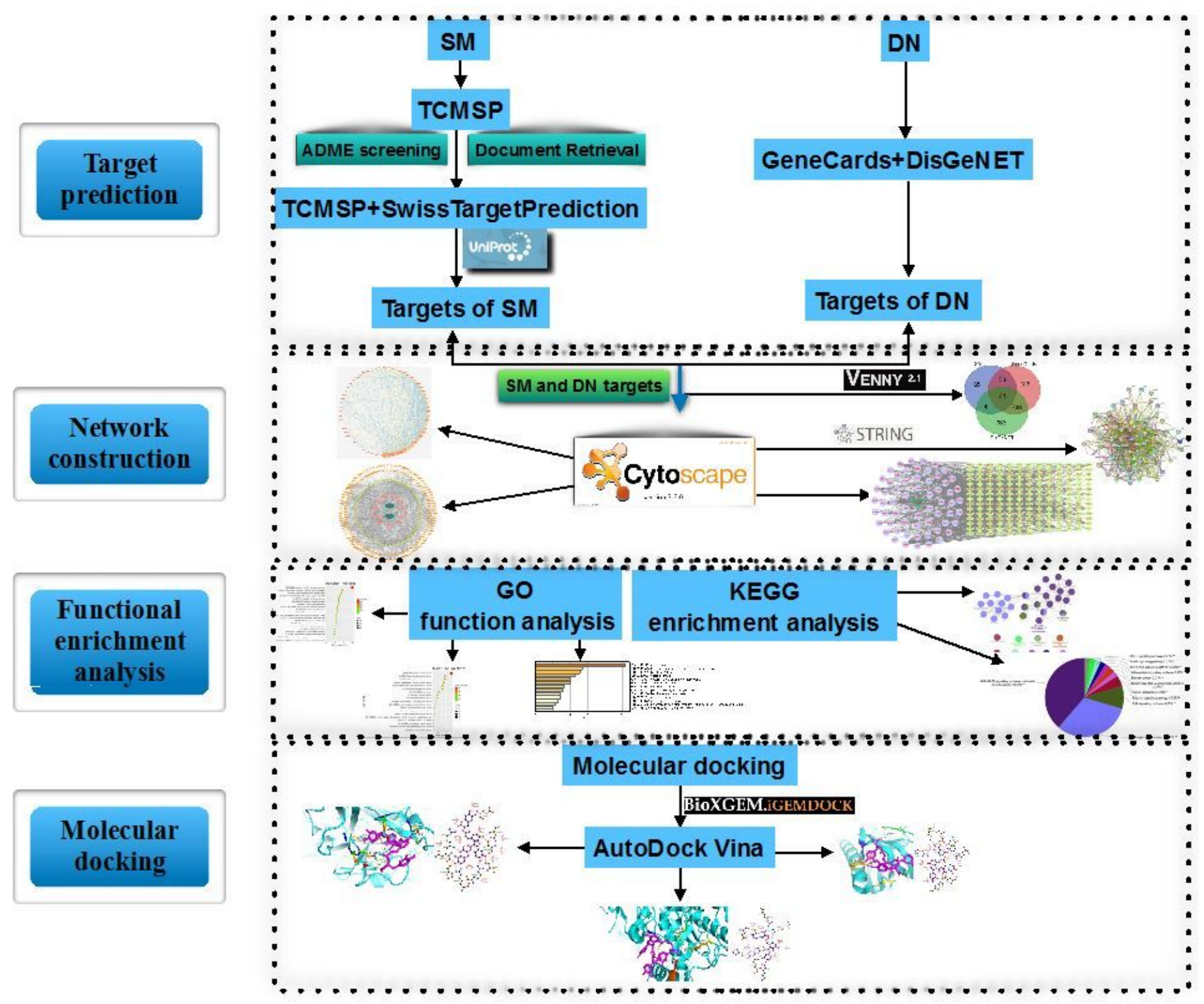

Figure 1

Flowchart based on network pharmacology and molecular docking. 


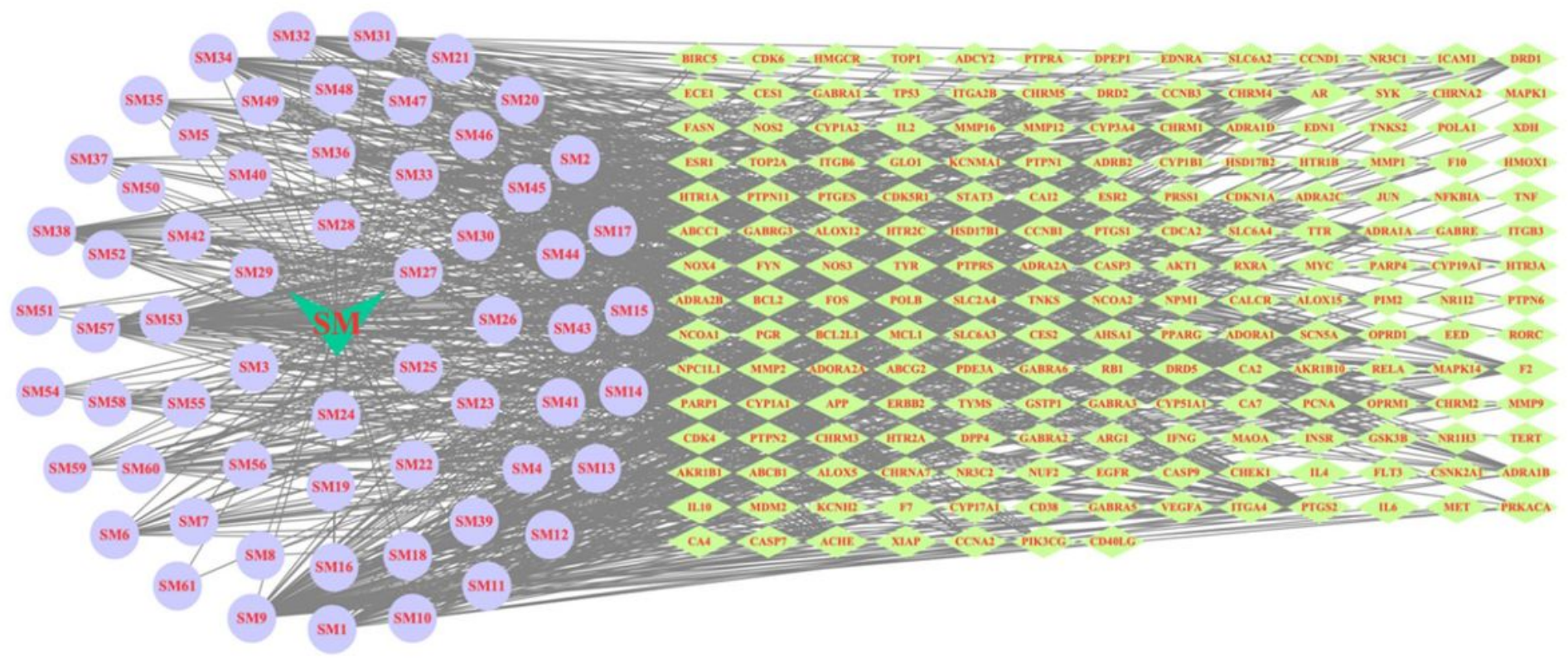

Figure 2

Network construction of "ingredients-targets". The light cyan prism nodes represent the targets $\bowtie$ and the light purple round nodes represent the ingredients in SM.

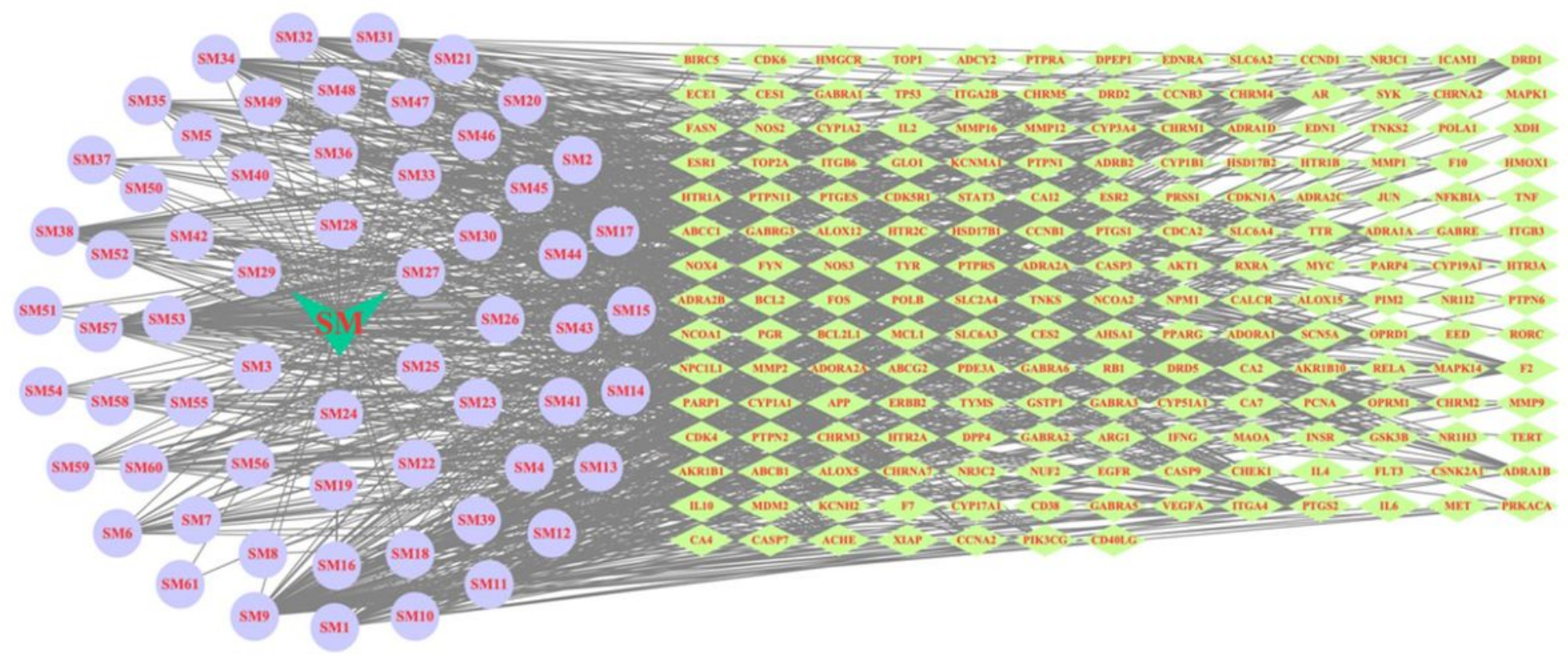

Figure 2

Network construction of "ingredients-targets". The light cyan prism nodes represent the targets $₫ a n d$ the light purple round nodes represent the ingredients in SM. 


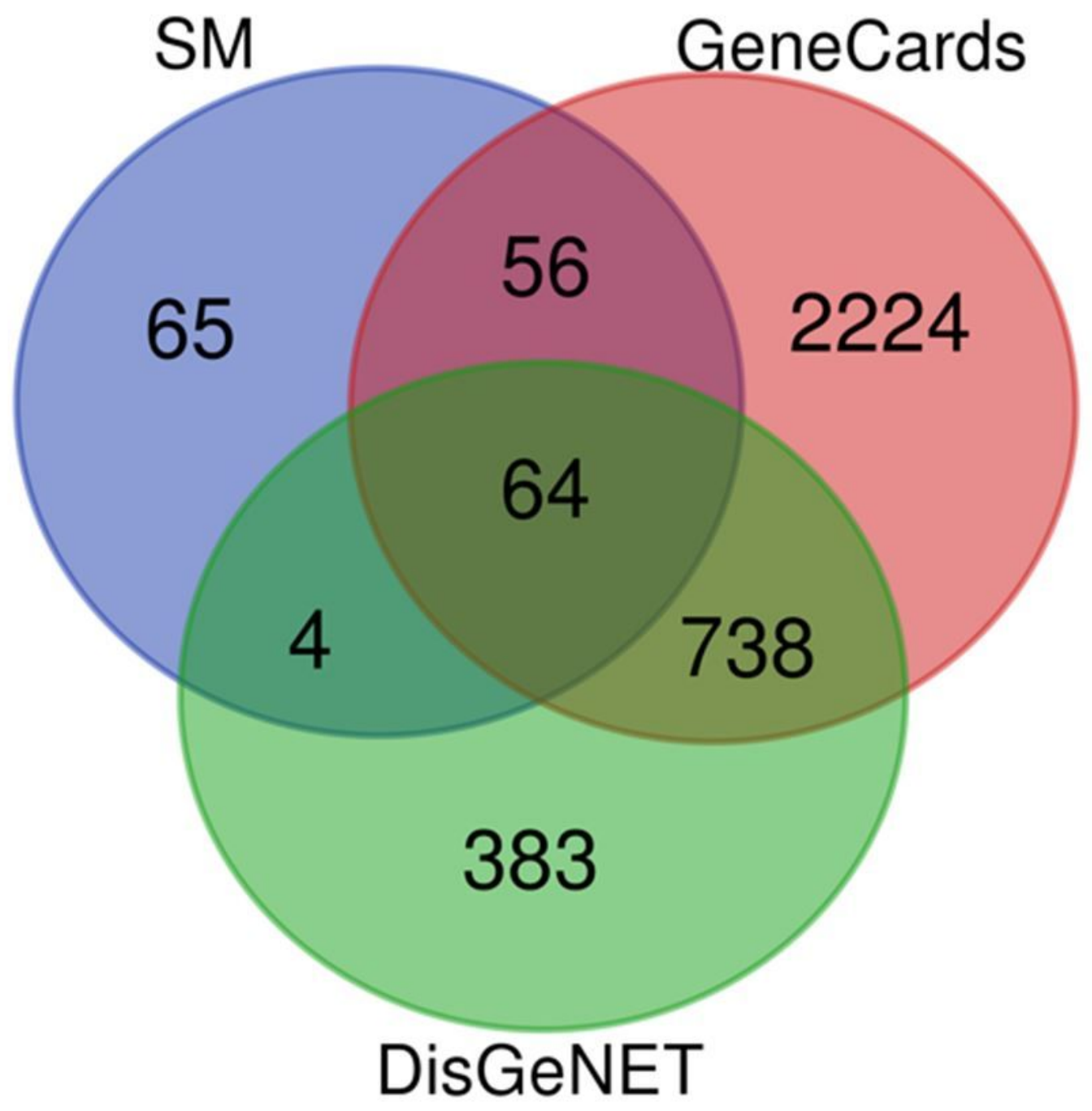

Figure 3

SM-DN common target genes. 


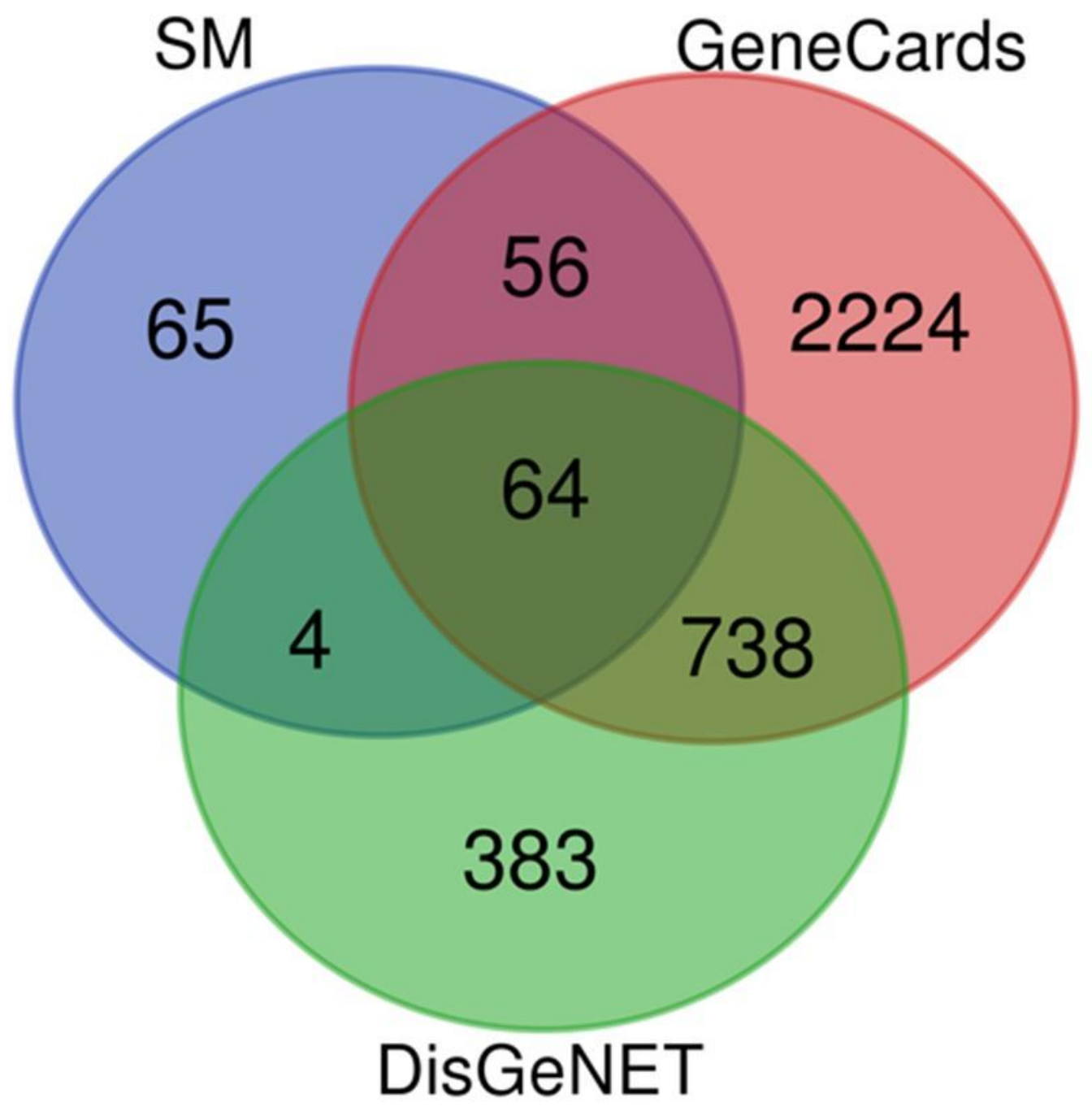

Figure 3

SM-DN common target genes. 
A
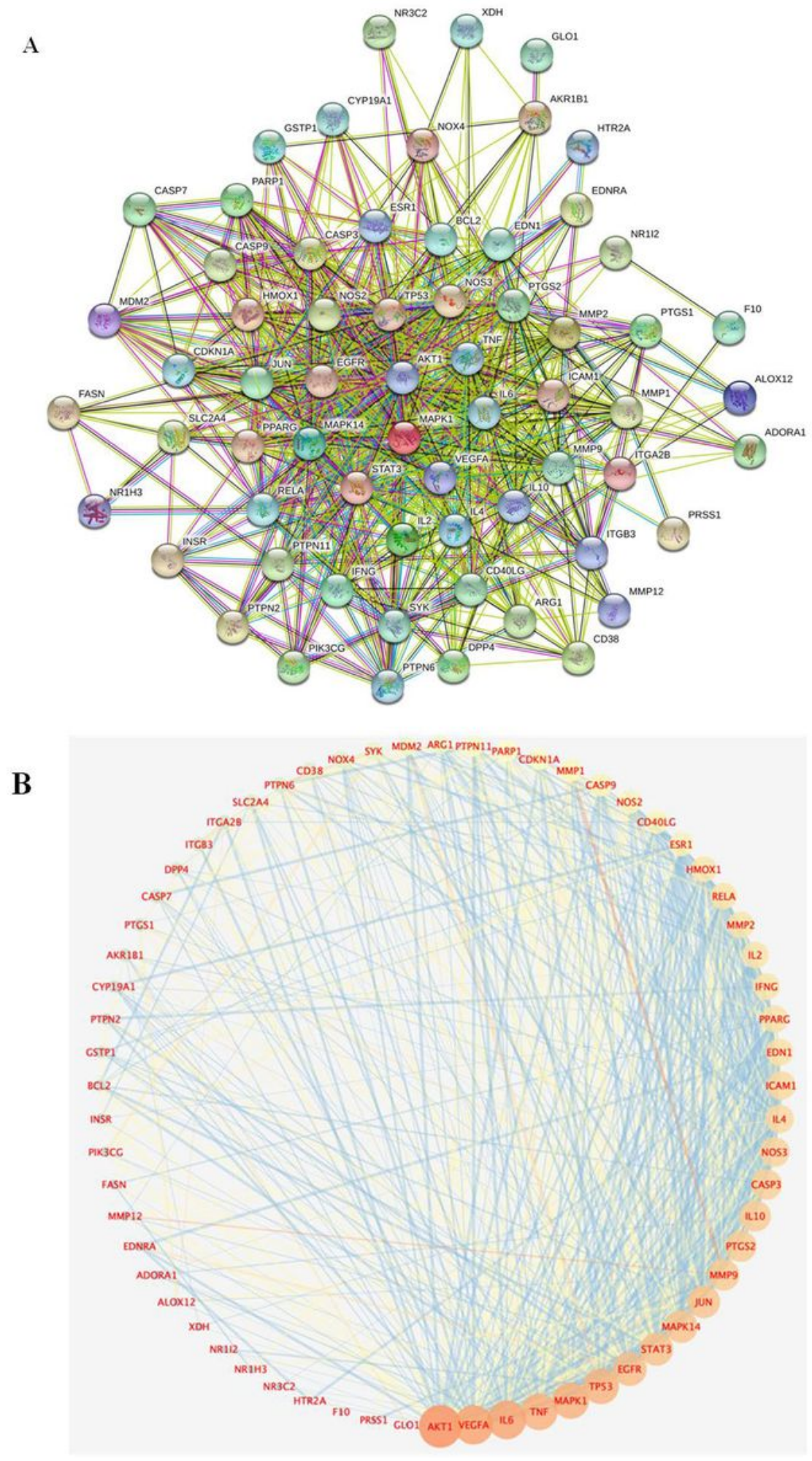

\section{Figure 4}

PPI network analysis. A: PPI networks of targets by STRING 11.0. Nodes represent proteins. Edges represent protein-protein associations. B: Potential target genes are arranged counterclockwise according to the degree value from large to small. 
A
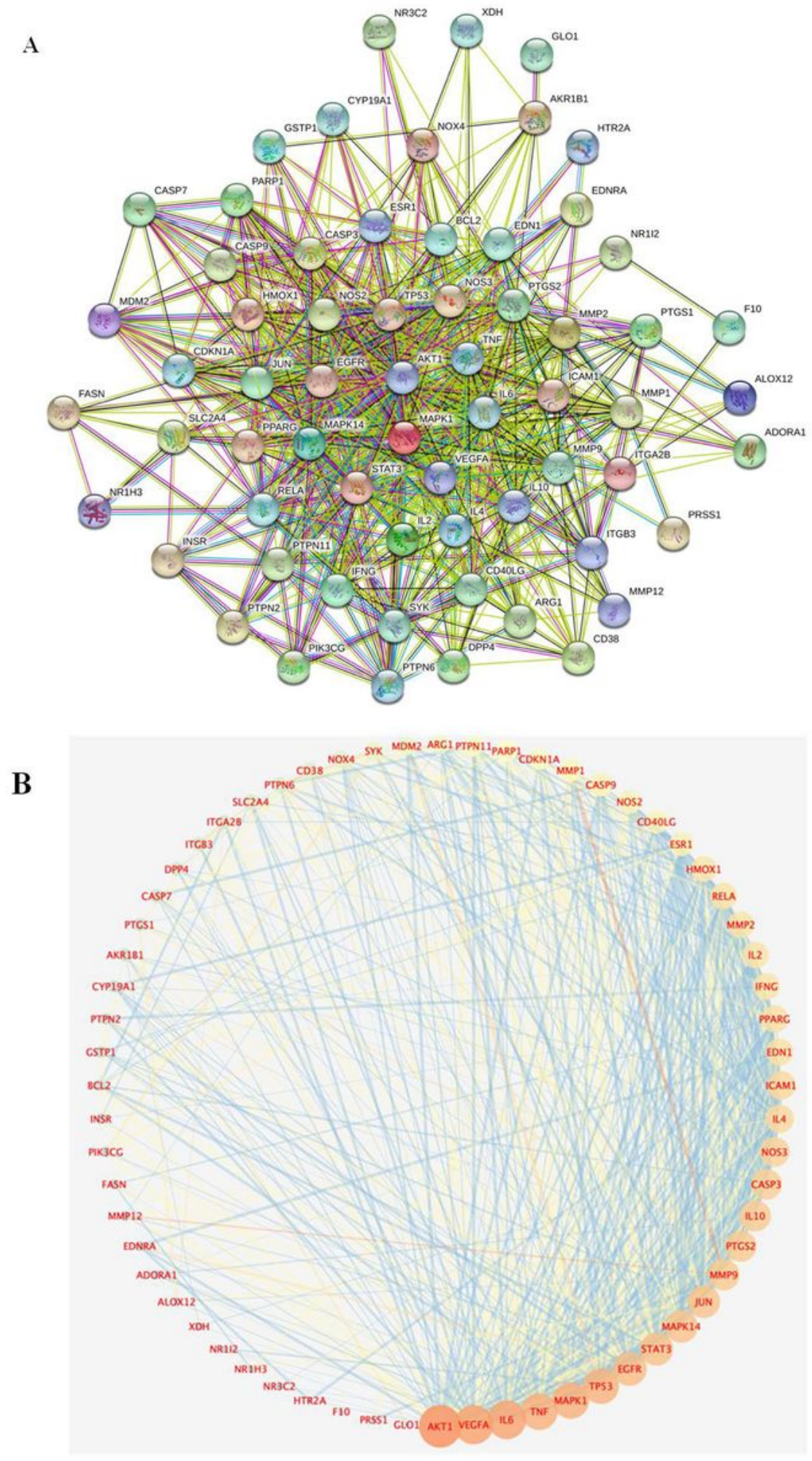

\section{Figure 4}

PPI network analysis. A: PPI networks of targets by STRING 11.0. Nodes represent proteins. Edges represent protein-protein associations. B: Potential target genes are arranged counterclockwise according to the degree value from large to small. 
A

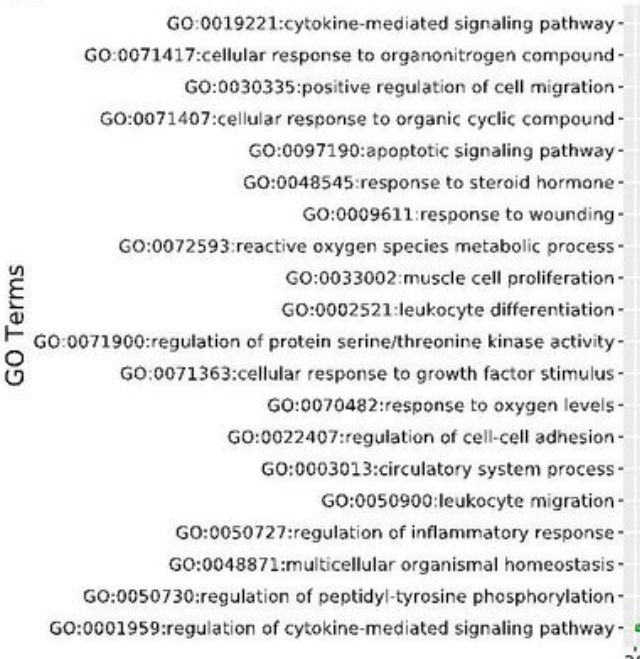

19221:cytokine-mediated signaling pathway:cellular response to organonitrogen compoundlular response to organic cyclic compoundG0:0097190:apoptotic signaling pathwayesponse to steroid hormoneexygen species metabolic process G0:0033002:muscle cell proliferationsponse to growth factor stimulus 022407:regulation of cell-cell adhesion 003013 :circulatory system process GO:0050900:leukocyte migration GO:0050727:regulation of inflammatory response GO:0048871:multicellular organismal homeostasis osphorylation 20 protein serine/threonine kinase activity-

\section{Biological Processes}

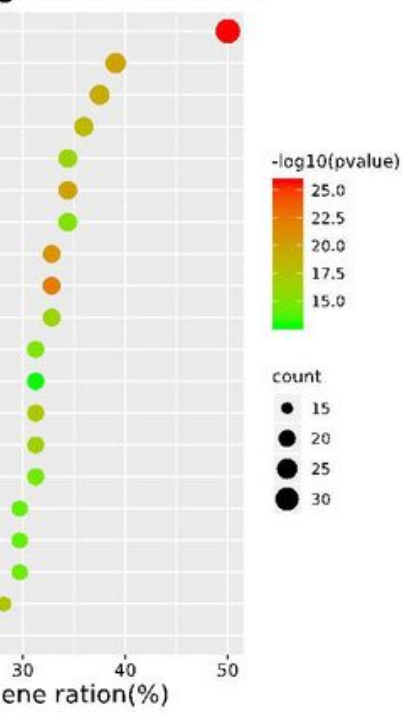

B

\section{Molecular Functions}

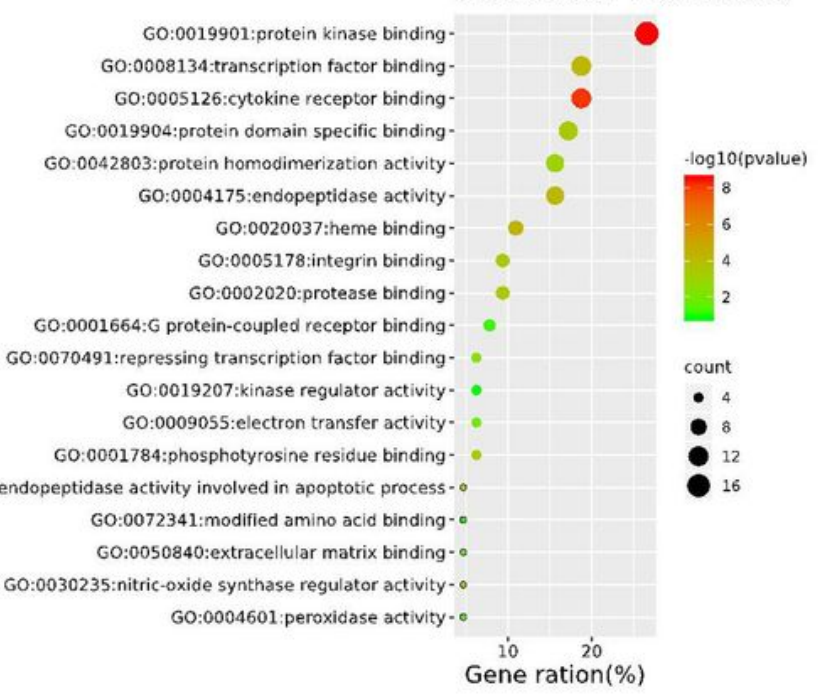

C

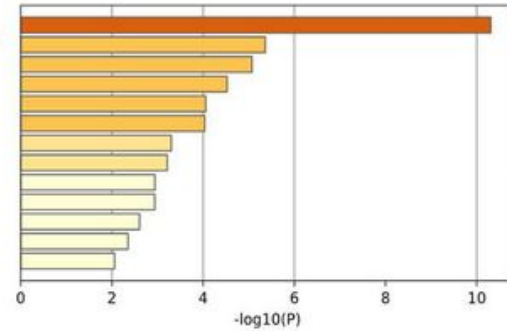

GO:0045121: membrane raft rase II transcription factor complex GO:0043235: receptor complex GO:0031983: vesicle lumen 60:0009897: external side of plasma membrane 60:0005912: adherens junction 60:0031012: extracellular matrix 60:0048471: perinuclear region of cytoplasm 60:0031256: leading edge membrant 60:0030139: endocytic vesicle

GO:0061695: transferase complex, transferring phosphorus-containing groups 60:0005788: endoplasmic reticulum lumen

\section{Figure 5}

GO enrichment analysis. These include (A) biological process (BP) terms, (B) molecular function (MF) terms (C) cellular component (CC) terms. A, B: The color of the node is displayed in a gradient from red to green according to the descending order of the $P$ value. The size of the nodes is arranged in ascending order of the number of genes. C: Sort by the importance of -Log10 (P) of each lane. 
A

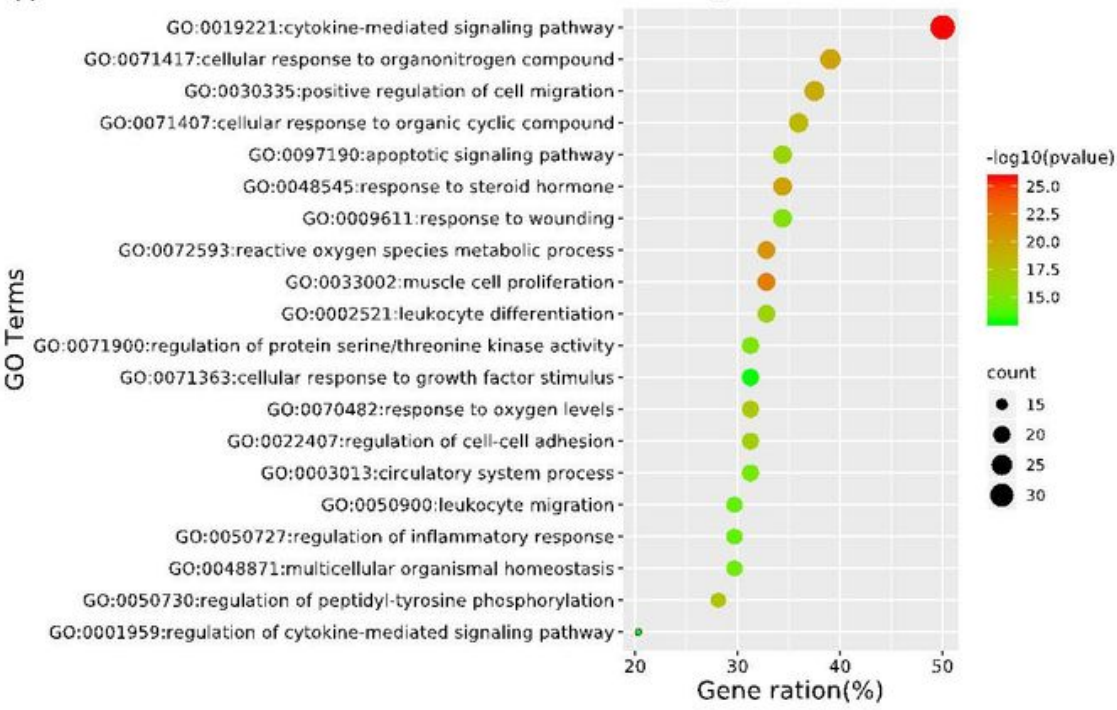

B

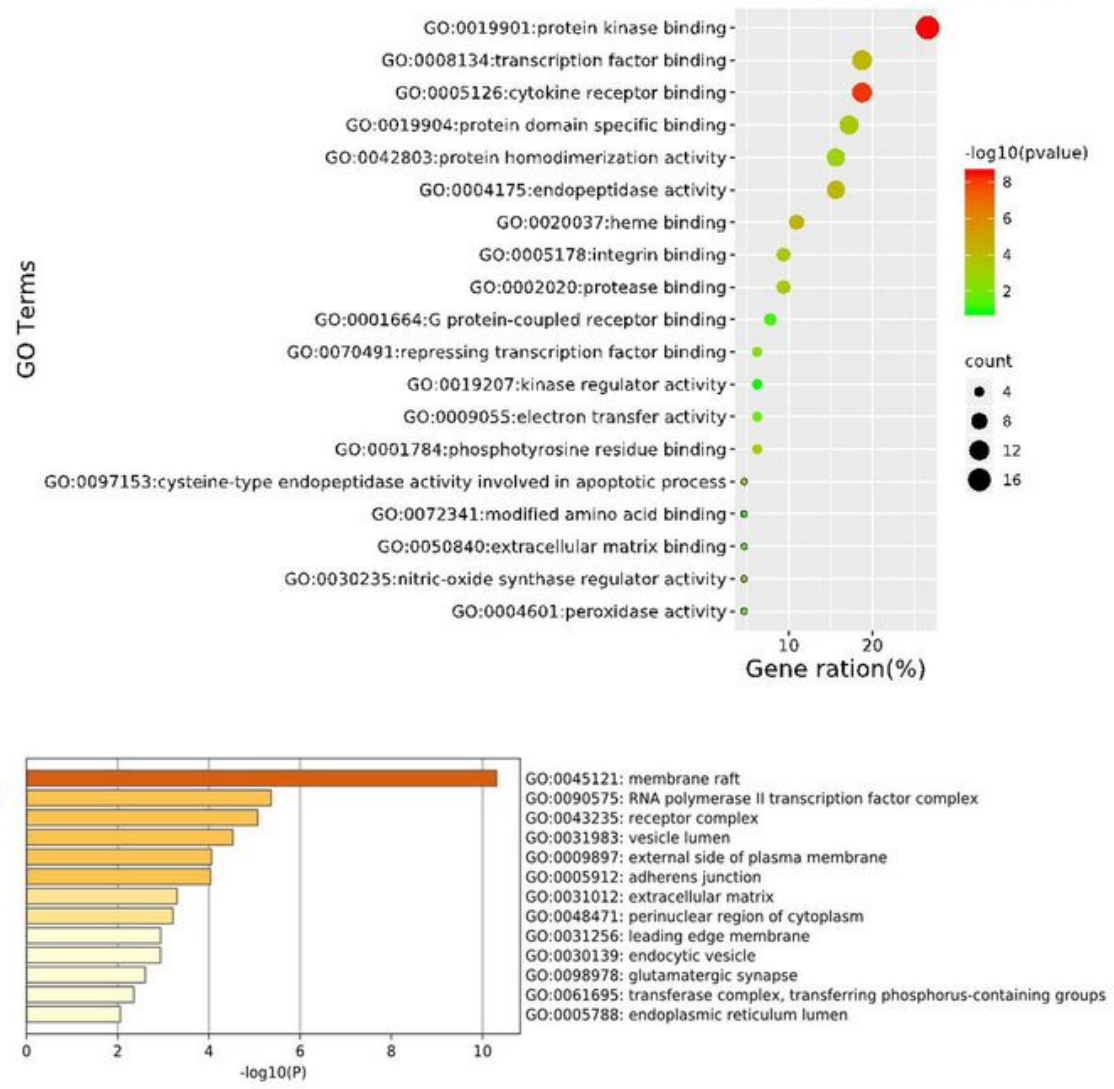

Figure 5

GO enrichment analysis. These include (A) biological process (BP) terms, (B) molecular function (MF) terms (C) cellular component (CC) terms. A, B: The color of the node is displayed in a gradient from red to green according to the descending order of the $P$ value. The size of the nodes is arranged in ascending order of the number of genes. C: Sort by the importance of -Log10 (P) of each lane. 

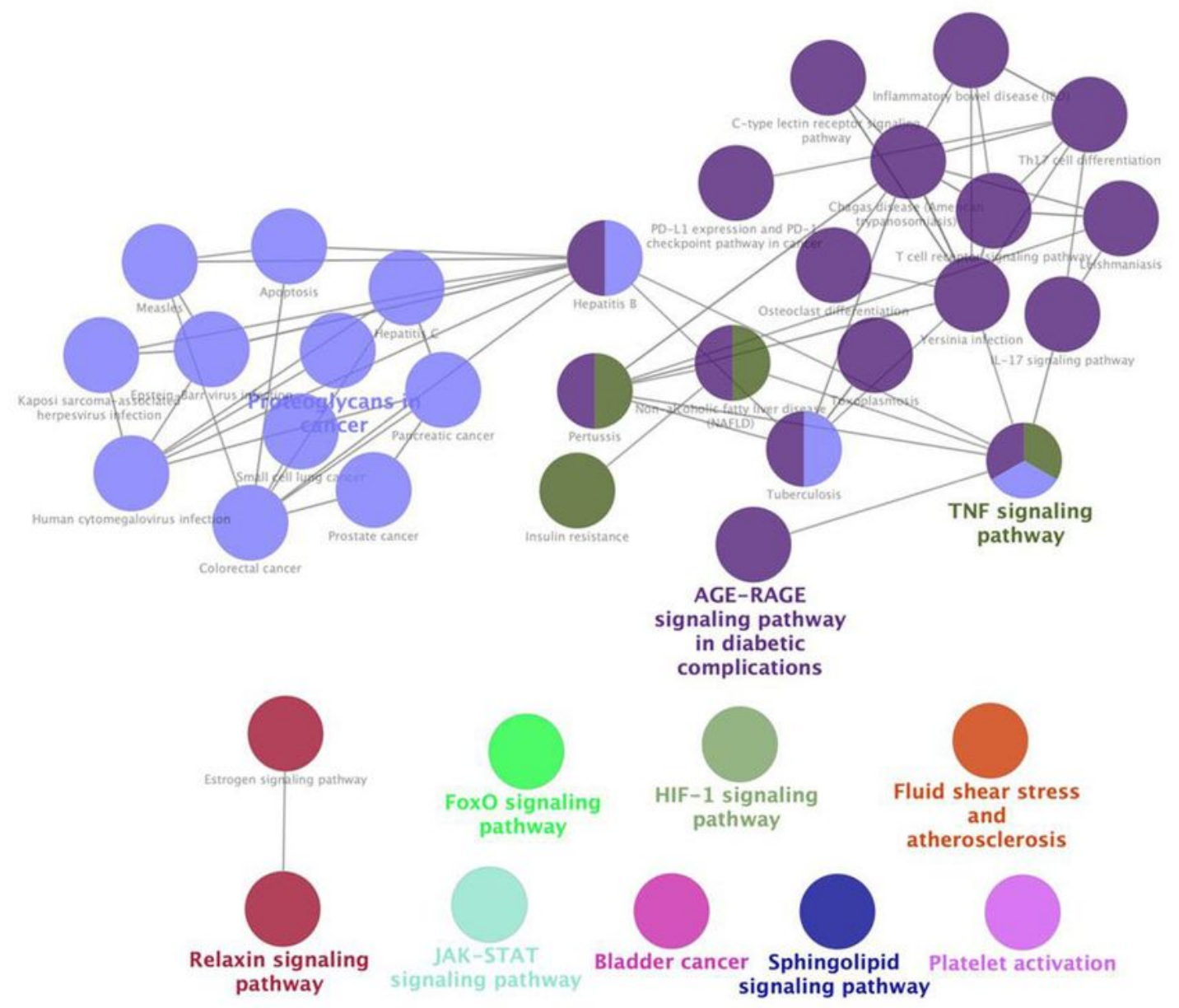

B

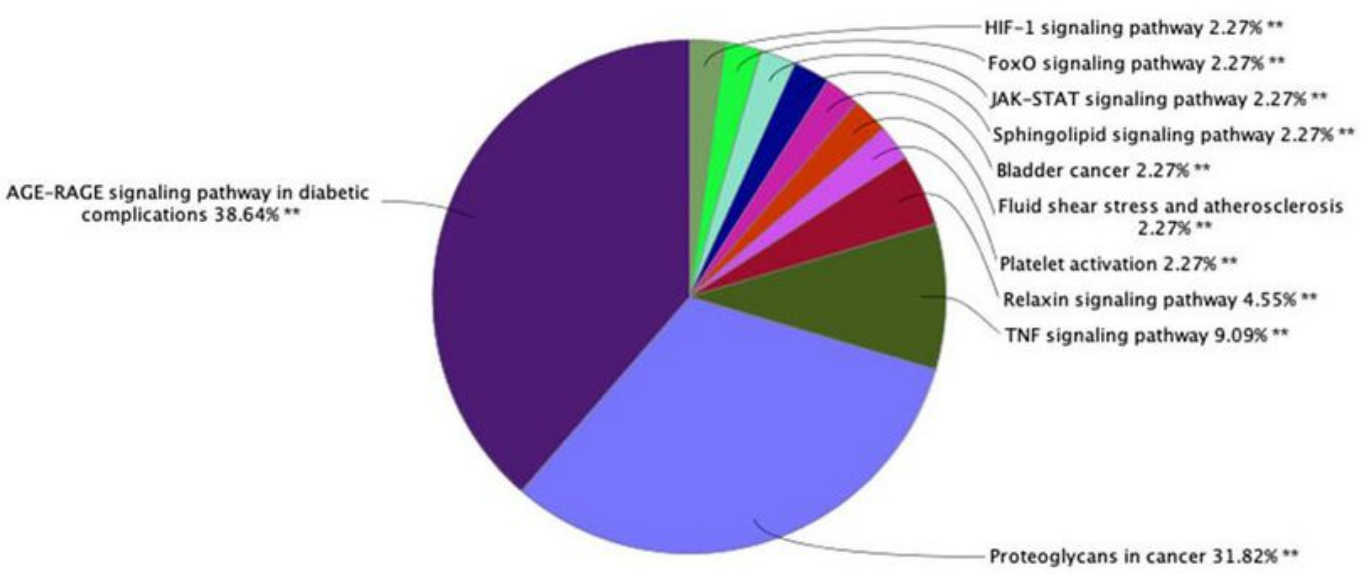

\section{Figure 6}

KEGG pathway analysis of potential targets of SM among DN-related proteins using the ClueGO plug-in. The KEGG term is expressed as a node, and the size of the node indicates importance. Only the most significant terms in the group have been labeled. 


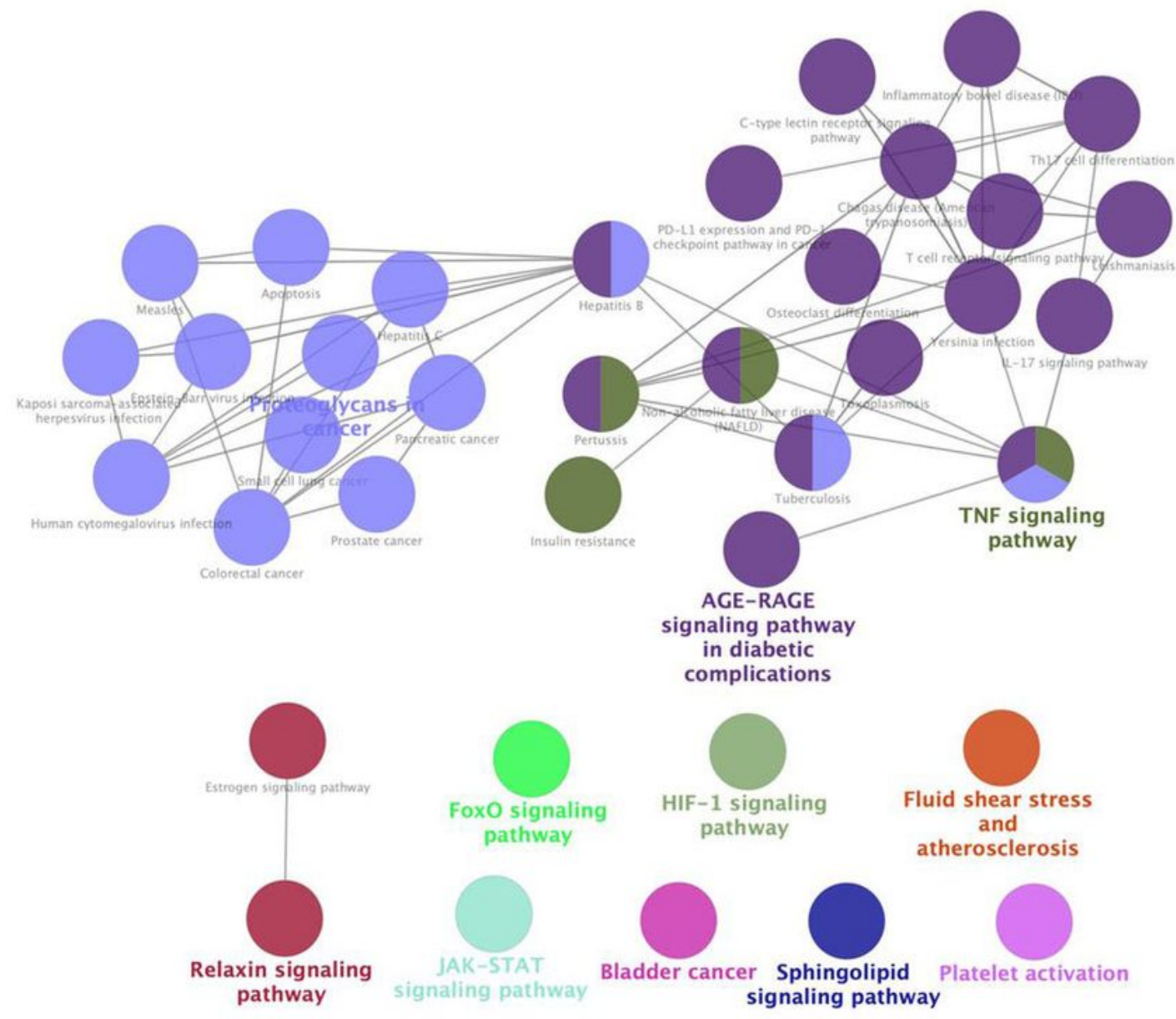

B

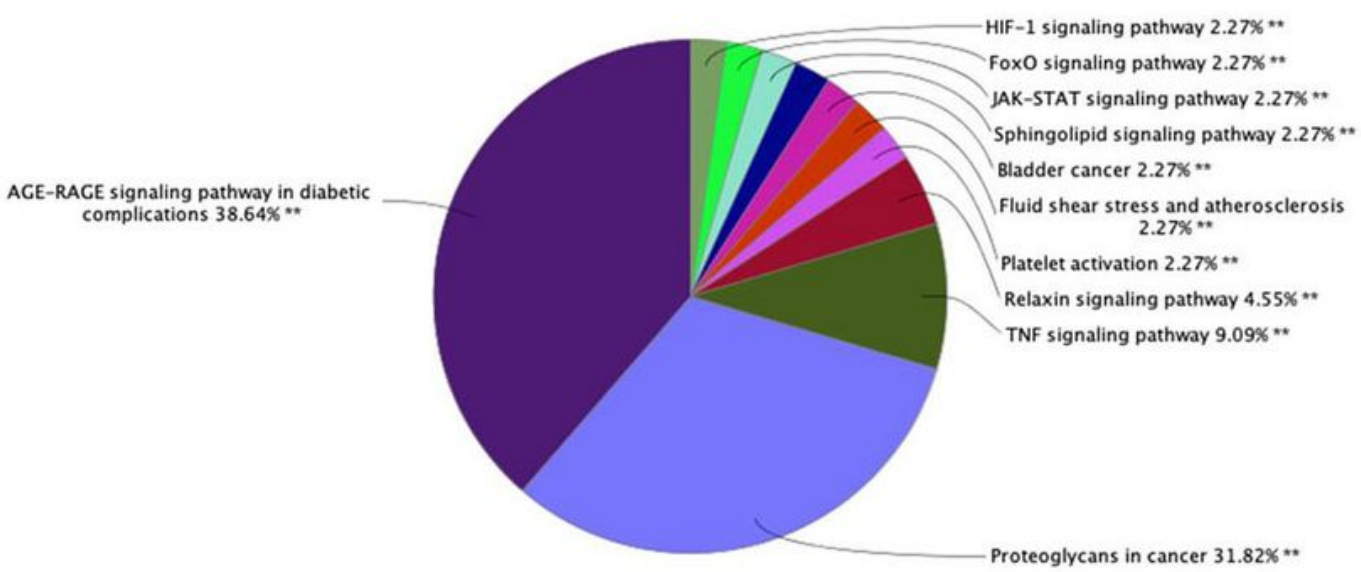

\section{Figure 6}

KEGG pathway analysis of potential targets of SM among DN-related proteins using the ClueGO plug-in. The KEGG term is expressed as a node, and the size of the node indicates importance. Only the most significant terms in the group have been labeled. 
A
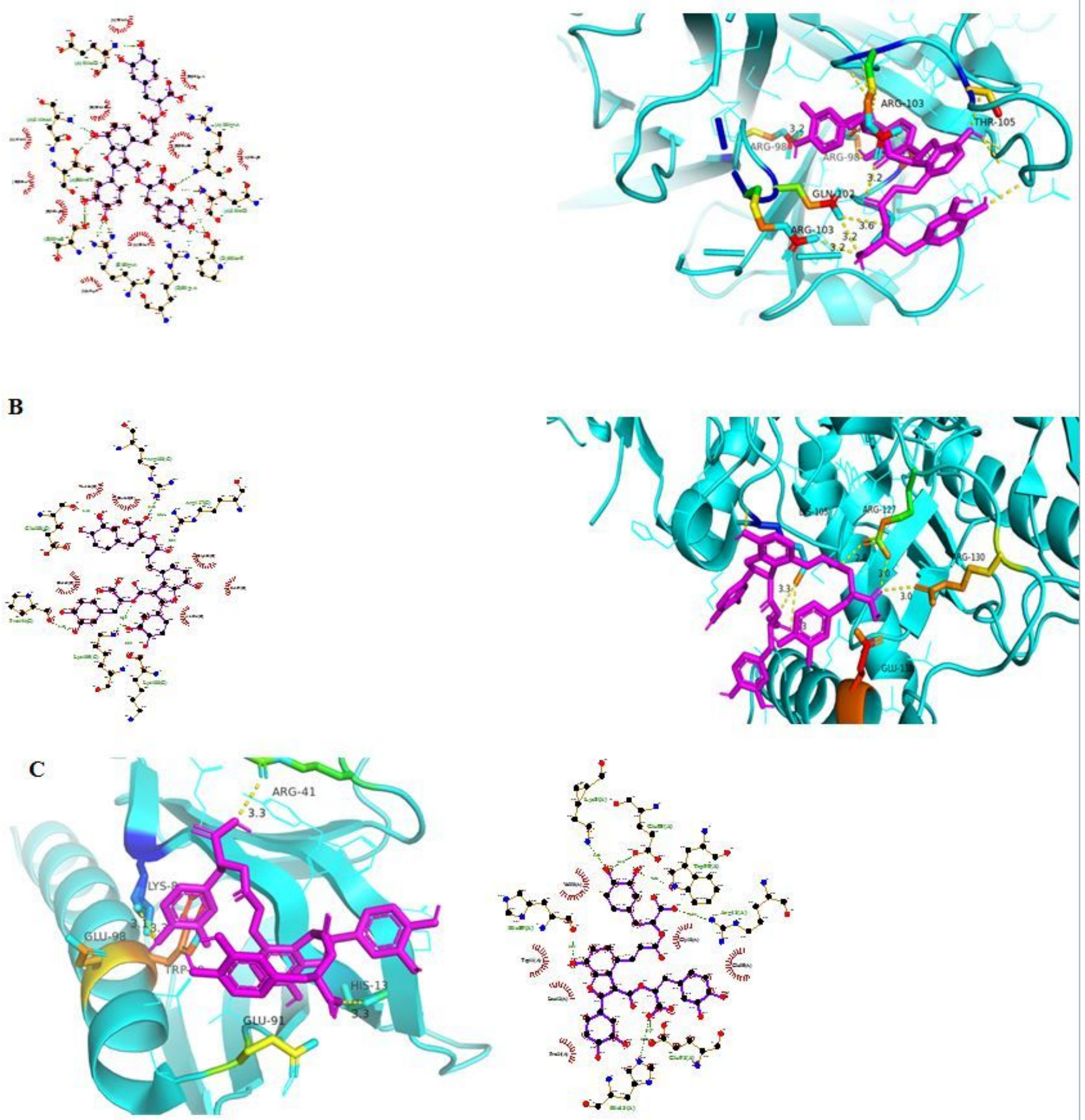

Figure 7

Molecular docking. Molecular models of the binding of salvianolic acid B with (A) TNF, (B) NOS2, and (C) AKT1 shown as 3D diagrams and 2D diagrams. 
A


Figure 7

Molecular docking. Molecular models of the binding of salvianolic acid B with (A) TNF, (B) NOS2, and (C) AKT1 shown as 3D diagrams and 2D diagrams. 


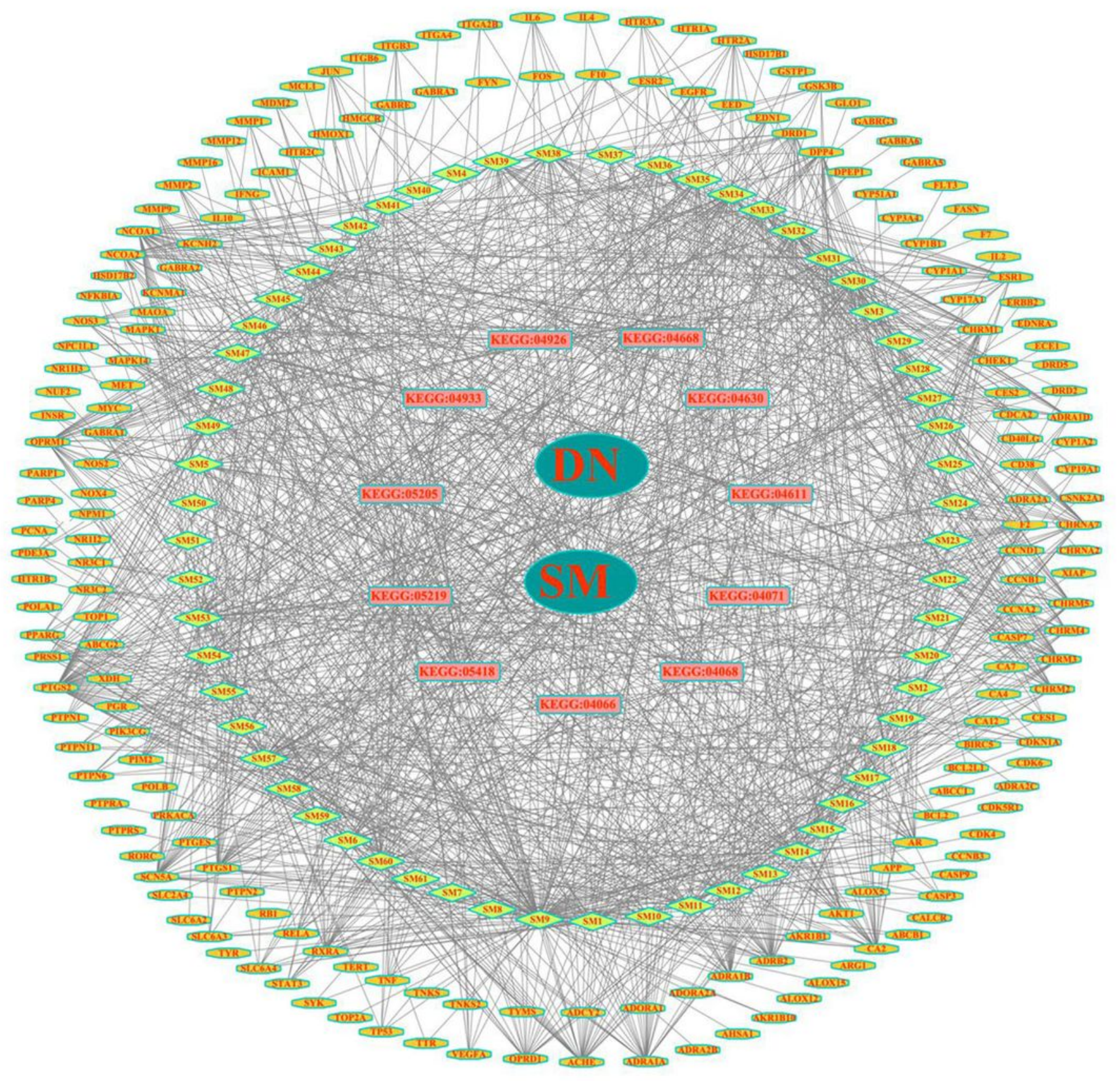

Figure 8

The network structure of "SM-component-target-pathway-DN". "Oval" represents SM and DN, "diamond" represents components, "hexagon" represents target, and "rectangle" represents pathway. 


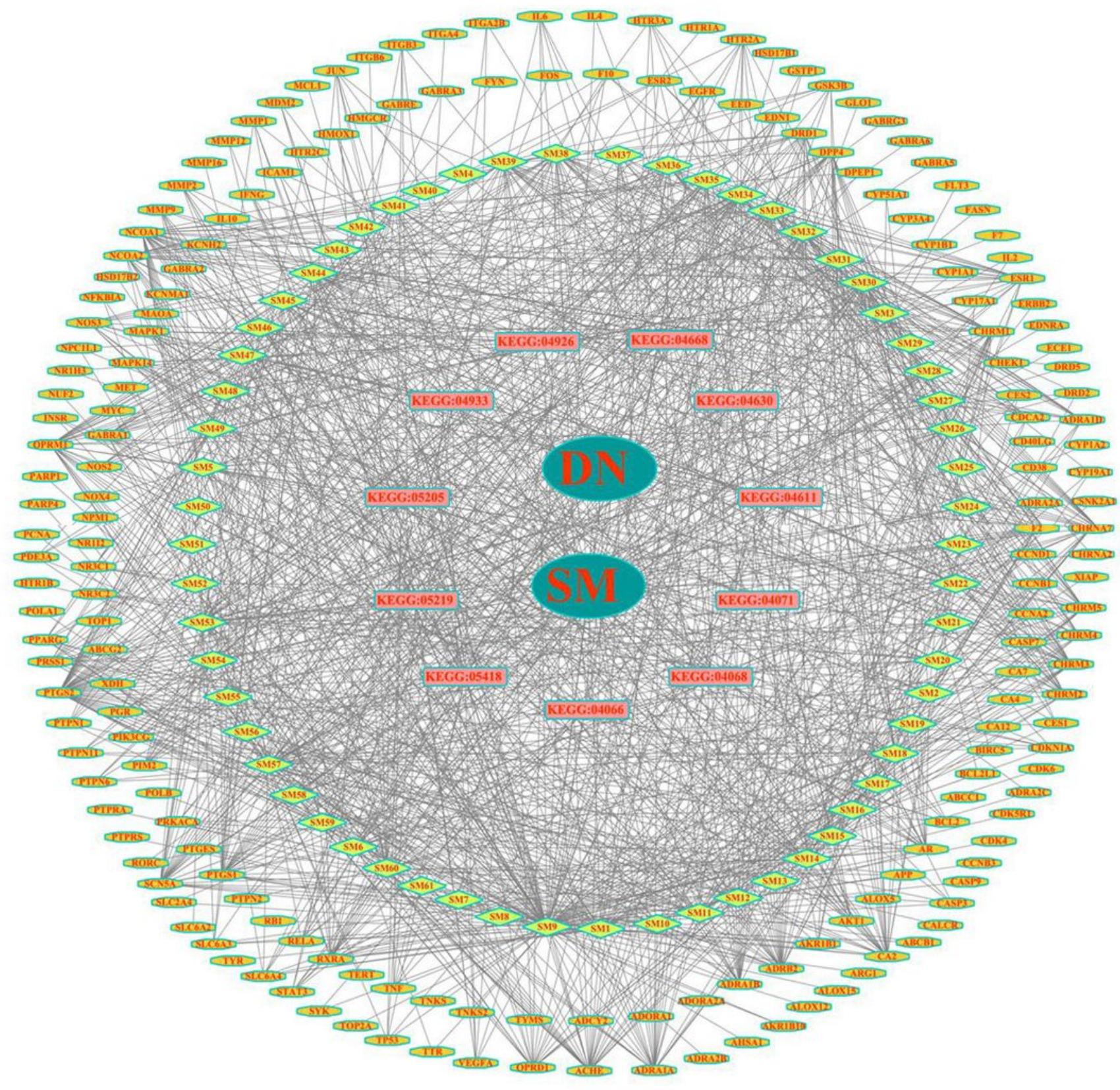

Figure 8

The network structure of "SM-component-target-pathway-DN". "Oval" represents SM and DN, "diamond" represents components, "hexagon" represents target, and "rectangle" represents pathway.

\section{Supplementary Files}

This is a list of supplementary files associated with this preprint. Click to download.

- Tables4.xls

- Tables4.xls 
- Tables3.xlsx

- TableS3.xIsx

- Tables2.xlsx

- Tables2.xlsx

- TableS1.xlsx

- Tables1.xlsx 